\title{
Robust Adaptive Control for Nonlinear Uncertain Systems Using Type-2 Fuzzy Neural Network System
}

\author{
Ching-Hung Lee and Yu-Ching Lin \\ Department of Electrical Engineering, Yuan Ze University, Chung-li, Taoyuan 320, Taiwan \\ Correspondence should be addressed to Ching-Hung Lee, chlee@saturn.yzu.edu.tw
}

Received 15 December 2010; Revised 19 March 2011; Accepted 6 April 2011

Academic Editor: Wei-Chiang Hong

Copyright (c) 2011 C.-H. Lee and Y.-C. Lin. This is an open access article distributed under the Creative Commons Attribution License, which permits unrestricted use, distribution, and reproduction in any medium, provided the original work is properly cited.

This paper proposes a novel intelligent control scheme using type-2 fuzzy neural network (type-2 FNN) system. The control scheme is developed using a type-2 FNN controller and an adaptive compensator. The type-2 FNN combines the type-2 fuzzy logic system (FLS), neural network, and its learning algorithm using the optimal learning algorithm. The properties of type-1 FNN system parallel computation scheme and parameter convergence are easily extended to type2 FNN systems. In addition, a robust adaptive control scheme which combines the adaptive type-2 FNN controller and compensated controller is proposed for nonlinear uncertain systems. Simulation results are presented to illustrate the effectiveness of our approach.

\section{Introduction}

In recent years, the fuzzy systems (FSs) and neural networks (NNs) have successfully been applied in nonlinear system identification and control [1-11]. The fuzzy neural network (FNN) system is realized with the FS in the NN structure. Thus, the FNN becomes an active subject in many areas due to its advantages, such as universal approximation, learning ability, and convergence of parameters $[2,8,12]$. The above advantages are established by training the parameters of FNN through iterations. In particular, the backpropagation (BP) algorithm (also known as gradient descent method) is usually adopted to tune the parameters of FNN, which consist of fuzzy sets and the weighting factors of NN $[2,8,12,13]$. For each of the iterations, all parameters of FNN are adjusted to reduce the error between the desired and actual outputs. The cost function is the indicator adopted to minimize the error. Therefore, the dynamic and optimal learning rate for FNN has been proposed to accelerate the convergence of the BP algorithm [3, 14-16].

To treat the "uncertainty information" problem, Zadeh proposed the concept of a type2 fuzzy system which is an extension of ordinary fuzzy sets (called type-1) [17]. Subsequently, 
Mendel and Karnik developed a complete theory of type-2 fuzzy logic systems (FLSs) $[7,10,18-20]$. These systems are characterized by IF-THEN rules, and type-2 fuzzy rules are more complex than type- 1 fuzzy ones because there are some differences, for example, their antecedents and consequent sets are type- 2 fuzzy sets $[7,10]$. By using type-2 fuzzy systems (T2 FSs), we outperform the use of type-1 fuzzy systems (T1 FSs). The T2FSs are described by type-2 fuzzy membership functions that are characterized by more design degrees of freedom $[19,21]$. This approach has been adopted in many applications, for example, system identification, nonlinear control, and signal processing [1, 4, 10, 14, 21-23].

In this paper, the interval valued type-2 fuzzy membership functions and interval sets are utilized to implement in the network structure, called type-2 fuzzy neural network (type-2 FNN). Thus, the Type-2 FNN has more design degrees of freedom to enhance the performance. The analysis and applications of type- 2 FNN are proposed. The type-2 FNN is a multilayered connectionist network for realizing the type-2 fuzzy inference system, and it can be constructed from a set of type-2 fuzzy rules. The type-2 FNN consists of type2 fuzzy linguistic process as both the antecedent and consequent parts. The consequent part denotes the output through type reduction and defuzzification. The computation of interval type- 2 FNN is more complex than that of type- 1 one. In addition, we show that the characteristics of the FNN, fuzzy inference, and convergence properties, can be extended to type-2 FNN. According to the Lyapunov theorem, rigorous proofs are presented to guarantee the convergence of type-2 FNN and system stability. An adaptive control scheme using type2 FNN is presented to treat the control problem of nonlinear uncertain system. Simulation results are shown to demonstrate the effectiveness and performance of the proposed type- 2 FNN system.

The paper is organized as follows. In Section 2, we briefly introduce the type-2 FNN system. Section 3 presents the main result of adaptive control scheme and optimal learning for type-2 FNN controller. In Section 4, the simulation results of the control uncertain chaotic system are presented. Concluding remarks are given in Section 5.

\section{Type-2 Fuzzy Neural Network Systems (Type-2 FNN)}

As the results of previous literature and applications, the fuzzy neural systems by using type-2 fuzzy systems (T2 FSs) can outperform the use of type-1 fuzzy systems (T1 FSs). The T2FSs are described by type- 2 fuzzy membership functions that are characterized by more design degrees of freedom $[19,21]$. Therefore, using T2 FSs has the potential to outperform using T1FSs, especially for uncertain environments. Herein, the interval valued type-2 fuzzy membership functions and interval sets are utilized to implement the type-2 fuzzy neural network (type-2 FNN). Details are introduced as follows.

\subsection{System Structure}

The construction of the $j$ th component of type- 2 FNN system is shown in Figure 1, which is a kind of fuzzy inference system in the neural network structure $[1-3,9,15]$. When compared with type-1 FNN, the major difference is that the type-1 fuzzy membership functions (MFs) are replaced by type- 2 ones and the interval sets of consequent part. Herein, we first indicate the signal propagation and the basic function of every node in each layer. In the following symbols, the subscript $i j$ indicates the $j$ th term of the $i$ th input $O_{i j}^{(k)}$, where $j=1, \ldots, l$, and the superscript $(k)$ denotes the $k$ th layer. 


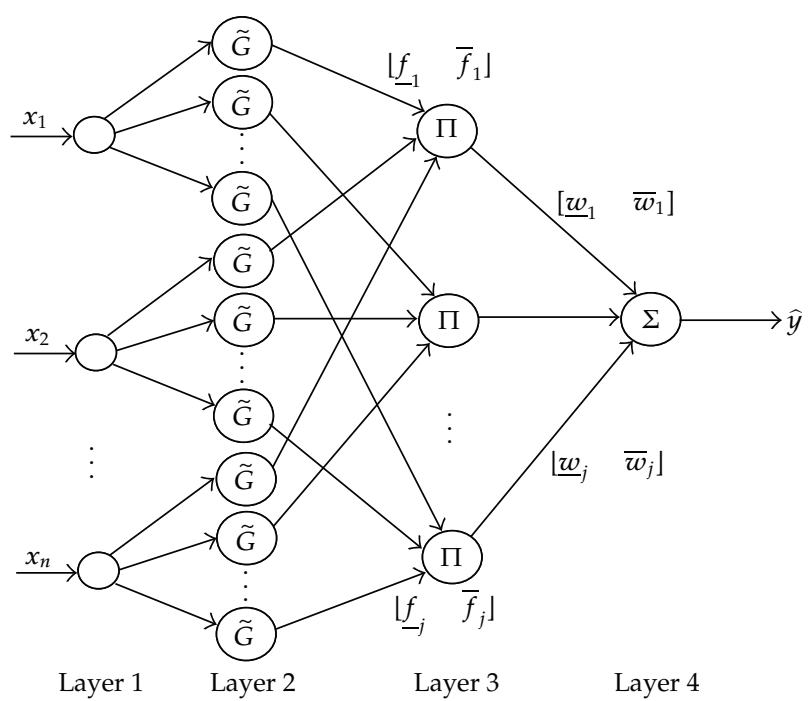

Figure 1: Construction of MISO type-2 FNN system with uncertain mean.

\section{Layer 1: Input Layer}

For the $i$ th node of layer 1 , the net input and output are represented as

$$
O_{i}^{(1)}=w_{i}^{(1)} x_{i}^{(1)},
$$

where the weights $w_{i}^{(1)}=1, i=1, \ldots, n$ and $x_{i}^{(1)}$ represent the $i$ th input to the $i$ th node of layer 1.

\section{Layer 2: Membership Layer}

In this layer, each node performs a type-2 interval fuzzy MF, as shown in Figure 2. Note that when all T2 FSs are interval type, then the firing set and fired rule output set are interval values, and this simplifies all computational effort enormously. We here introduce two cases of the output of layer $2[7,10,18-20]$.

Case 1. For the Gaussian MF with uncertain mean as shown in Figure 2(a)

$$
O_{i j}^{(2)}=\exp \left[-\frac{1}{2} \frac{\left(O_{i}^{(1)}-m_{i j}\right)^{2}}{\left(\sigma_{i j}\right)^{2}}\right]=\left\{\begin{array}{l}
\bar{O}_{i j}^{(2)} \text { as } m_{i j}=\bar{m}_{i j}, \\
\underline{O}_{i j}^{(2)} \text { as } m_{i j}=\underline{m}_{i j} .
\end{array}\right.
$$

Case 2. For the Gaussian MF with uncertain variance as shown in Figure 2(b)

$$
O_{i j}^{(2)}=\exp \left[-\frac{1}{2} \frac{\left(O_{i}^{(1)}-m_{i j}\right)^{2}}{\left(\sigma_{i j}\right)^{2}}\right]=\left\{\begin{array}{l}
\bar{O}_{i j}^{(2)} \text { as } \sigma_{i j}=\bar{\sigma}_{i j}, \\
\underline{O}_{i j}^{(2)} \text { as } \sigma_{i j}=\underline{\sigma}_{i j}
\end{array}\right.
$$




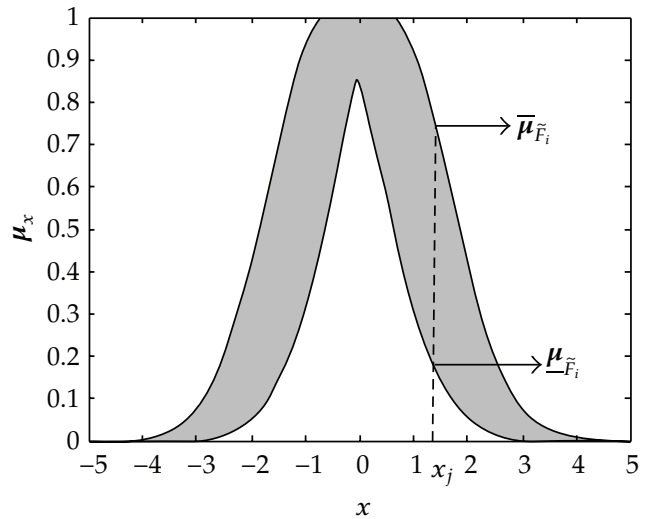

(a)

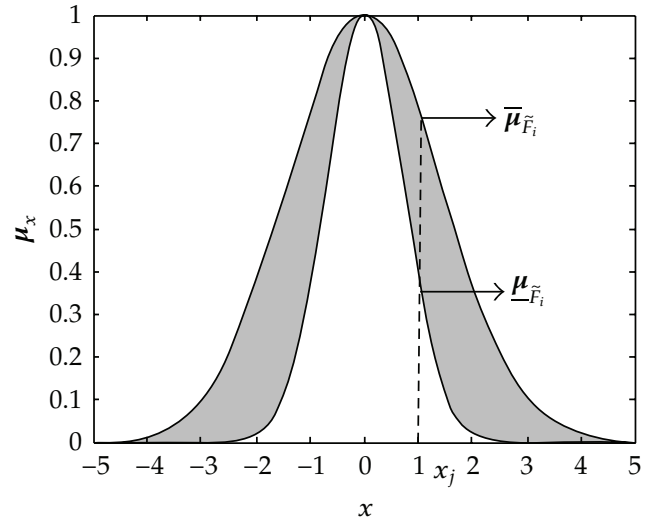

(b)

Figure 2: Gaussian MFs, (a) uncertain mean by upper and lower MFs; (b) uncertain variance by upper and lower MFs.

where $m_{i j}$ and $\sigma_{i j}$ represent the center (or mean) and the width (or variance), respectively. As shown in Figure 2, type-2 interval MFs can be represented as interval bound by upper and lower MFs, denoted by $\bar{\mu}_{\widetilde{F}_{i}}$ and $\underline{\mu}_{\widetilde{F}_{i}}$, respectively. Therefore, the output $O_{i j}^{(2)}$ is represented as $\left[\underline{O}_{i j}^{(2)}, \bar{O}_{i j}^{(2)}\right]$.

\section{Layer 3: Rule Layer}

The links in this layer are employed to implement the antecedent matching, and they work like rule engine of the type-2 FLSs. Here, the operation chosen is the simple PRODUCT operation. Then, for the $j$ th input rule node

$$
O_{j}^{(3)}=\prod_{i=1}^{n}\left(w_{i j}^{(3)} O_{i j}^{(2)}\right)=\left\{\begin{array}{l}
\bar{O}_{j}^{(3)}=\prod_{i=1}^{n}\left(w_{i j}^{(3)} \bar{O}_{i j}^{(2)}\right), \\
\underline{O}_{j}^{(3)}=\prod_{i=1}^{n}\left(w_{i j}^{(3)} \underline{O}_{i j}^{(2)}\right),
\end{array}\right.
$$

where the weights $w_{i j}^{(3)}$ are set to be unity. Similar to layer 2, the output $O_{i j}^{(3)}$ is represented as $\left[\underline{O}_{j}^{(3)}, \bar{O}_{j}^{(3)}\right]$.

\section{Layer 4: Output Layer}

The links in this layer are employed to implement the consequent matching, type reduction, and defuzzification $[7,10,14,18-20]$. A type-reducer combines all fired-rule output sets in some way, just like a type-2 defuzzifier combines the type- 1 rule output sets, which leads to a T1 FS that is called a type-reduced set. Finally, we defuzzify the type-reduced set to get a crisp output, that is,

$$
\widehat{y}=O^{(4)}=\frac{O_{R}^{(4)}+O_{L}^{(4)}}{2}
$$


where

$$
\begin{aligned}
& O_{R}^{(4)}=\sum_{j=1}^{l}\left(f_{j}^{R} \bar{w}_{j}^{(4)}\right)=\sum_{j=1}^{R}\left(\underline{O}_{j}^{(3)} \bar{w}_{j}^{(4)}\right)+\sum_{k=R+1}^{l}\left(\bar{O}_{j}^{(3)} \bar{w}_{k}^{(4)}\right), \\
& O_{L}^{(4)}=\sum_{j=1}^{l}\left(f_{j}^{L} \underline{w}_{j}^{(4)}\right)=\sum_{j=1}^{L}\left(\bar{O}_{j}^{(3)} \underline{w}_{j}^{(4)}\right)+\sum_{k=L+1}^{l}\left(\underline{O}_{j}^{(3)} \underline{w}_{k}^{(4)}\right) .
\end{aligned}
$$

According to the results of $[2,3]$, normalization is not used here. This simplifies the computation of type-2 FNN system in real-time applications. Moreover, in order to obtain $O_{L}^{(4)}$ and $O_{R}^{(4)}$, we need to find coefficients $R$ and $L$ first by the so-called Karnik-Mendel procedure $[10,19,20]$. Without loss of generality, it is assumed that the precomputed $\bar{w}_{j}^{(4)}$ and $\underline{w}_{j}^{(4)}$ are arranged in the ascending order, that is, $\bar{w}_{1}^{(4)} \leq \bar{w}_{2}^{(4)} \leq \cdots \leq \bar{w}_{l}^{(4)}$ and $\underline{w}_{1}^{(4)} \leq \underline{w}_{2}^{(4)} \leq \cdots \leq \underline{w}_{l}^{(4)}[7,10,20]$. The usual utilized Karnik-Mendel procedure for type reduction is introduced as follows:

$\mathrm{R}^{1}$ : compute $O_{R}^{(4)}$ in (2.6) by initially setting $f_{j}^{R}=1 / 2\left(\bar{O}_{j}^{(3)}+\underline{O}_{j}^{(3)}\right)$ for $i=1, \ldots, l$, and let $y_{r} \equiv O_{R}^{(4)}$;

$R^{2}$ : find $R(1 \leq R \leq l-1)$ such that $\bar{w}_{R}^{(4)} \leq y_{r} \leq \bar{w}_{R+1}^{(4)}$;

$\mathrm{R}^{3}$ : compute $O_{R}^{(4)}$ in (2.6) with $f_{j}^{R}=\underline{O}_{j}^{(3)}$ for $j \leq R$ and $f_{j}^{R}=\bar{O}_{j}^{(3)}$ for $j>R$, and let $y_{r}^{\prime}=O_{R}^{(4)}$;

$\mathrm{R}^{4}$ : if $y_{r}^{\prime} \neq y_{r}$, then go to step $\mathrm{R}^{5}$. If $y_{r}^{\prime}=y_{r}$, then stop and set $O_{R}^{(4)}=y_{r}^{\prime}$;

$\mathrm{R}^{5}$ : set $y_{r}^{\prime}$ equal to $y_{r}$, and return to step $\mathrm{R}^{2}$.

Subsequently, the computation of $O_{L}^{(4)}$ is similar to the above procedure. Thus, the input/output representation of type-2 FNN system with uncertain mean is

$$
\begin{aligned}
\widehat{y}\left(\bar{m}_{i j}, \underline{m}_{i j} \sigma_{i j}, \bar{w}_{j}, \underline{w}_{j}\right) \\
\quad=\frac{1}{2}\left[\sum_{j=1}^{R}\left(\underline{O}_{j}^{(3)} \bar{w}_{j}^{(4)}\right)+\sum_{k=R+1}^{l}\left(\bar{O}_{k}^{(3)} \bar{w}_{k}^{(4)}\right)+\sum_{j=1}^{L}\left(\bar{O}_{j}^{(3)} \underline{w}_{j}^{(4)}\right)+\sum_{k=L+1}^{l}\left(\underline{O}_{k}^{(3)} \underline{w}_{k}^{(4)}\right)\right] .
\end{aligned}
$$

Thus, the adjustable parameters of type-2 FNN system with uncertain mean are $\bar{m}, \underline{m}, \sigma, \bar{w}$, and $\underline{w}$. Furthermore, the type-2 MFs with uncertain variance, as shown in Figure 2(b), can be simplified as

$$
\widehat{y}\left(m_{i j}, \bar{\sigma}_{i j}, \underline{\sigma}_{i j}, w_{j}\right)=\frac{1}{2} \sum_{j=1}^{l}\left[\left(\underline{O}_{j}^{(3)}+\bar{O}_{j}^{(3)}\right) w_{j}^{(4)}\right] .
$$

The adjustable parameters of type-2 FNN system with uncertain variance are $m, \bar{\sigma}, \underline{\sigma}$, and $w$. Therefore, when the rule number is $R$, the parameters number of $n$ input and one output type- 2 FNN systems are $(3 n+2) \times R$ and $(3 n+1) \times R$ for uncertain mean 


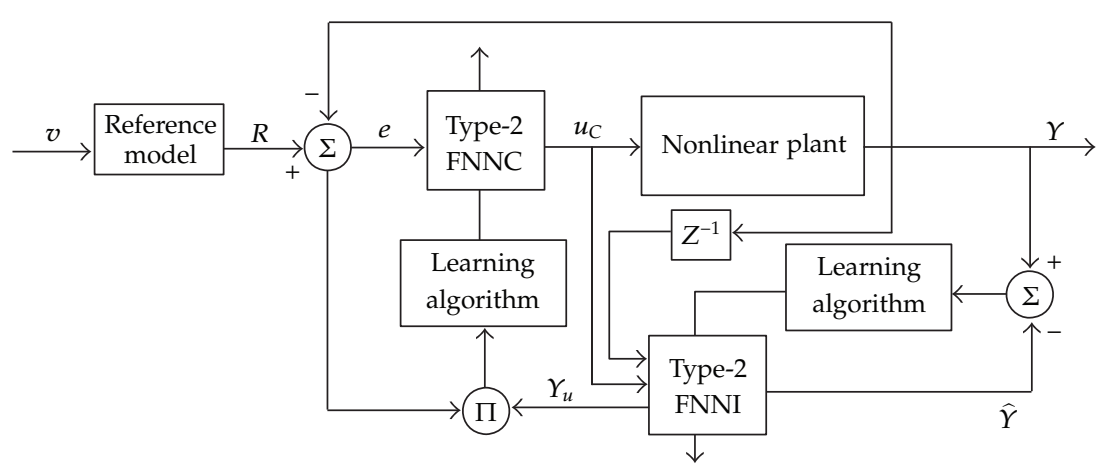

Figure 3: Adaptive control scheme using type-2 FNNs.

and variance, respectively. As the same condition, the type- 1 FNN system has $(2 n+1) \times R$ adjustable parameters $(m, \sigma$, and $w)$.

\subsection{Adaptive Control Scheme and Learning Algorithm}

According to the results of $[2,3]$, the model reference adaptive schematic of type-2 FNN control system is shown in Figure 3. The control objective of the nonlinear plant is to make the system output $Y$ follow the reference input $R$ and minimize the system error $e$. The purpose of the control scheme is to use the system control error $e=Y-R$ through the type-2 FNN controller (type-2 FNNC) to generate the proper control signal $u_{C}$. In order to minimize the system error, the weights of type-2 FNN are updated on line through the dynamic gradient descent learning algorithm. Let the cost function be minimized, which is defined as

$$
E_{C}=\frac{1}{2}(R-Y)^{2}
$$

That is, our goal is to minimize the tracking error. For training type-2 FNN, we utilize the well-known backpropagation algorithm with time-varying learning rate $[4,14,15,24]$. It can be written as

$$
W(k+1)=W(k)+\Delta W=W(k)+\eta(k)\left(-\frac{\partial E_{C}(k)}{\partial W}\right)
$$

where $\eta(k)$ and $W=[m, \bar{\sigma}, \underline{\sigma}, w]$ represent the time-varying learning rate and tuning parameters of type-2 FNN with uncertain variance, respectively. To obtain on line performance, avoid local minimum, and guarantee system stability, we use the Lyapunov theory to derive an adaptive learning algorithm with a time-varying learning rate to speed up the convergence. From (2.10) and (2.11), we have

$$
\Delta W=\eta(k)\left(-\frac{\partial E_{c}}{\partial W}\right)=\eta(k)\left(-\frac{\partial E_{c}}{\partial Y} \frac{\partial Y}{\partial u} \frac{\partial u}{\partial \widehat{y}} \frac{\partial \widehat{y}}{\partial W}\right)=-\eta(k)(R-Y) Y_{u} \frac{\partial \widehat{y}}{\partial W^{\prime}}
$$


where $Y_{u} \equiv \partial Y / \partial u$ denotes the system sensitivity and $\hat{y}$ denotes the type-2 FNN controller's output. The updated laws are represented as

$$
\begin{gathered}
m_{i j}=m_{i j}+\Delta m_{i j}, \quad w_{j}^{(4)}=w_{j}^{(4)}+\Delta w_{j}^{(4)}, \\
\bar{\sigma}_{i j}=\bar{\sigma}_{i j}+\Delta \bar{\sigma}_{i j}, \quad \underline{\sigma}_{i j}=\underline{\sigma}_{i j}+\Delta \underline{\sigma}_{i j} .
\end{gathered}
$$

Subsequently, we obtain the time-varying learning rate for the parameters using the results of [2-4]. We then have the following theorem.

Theorem 2.1. The type-2 FNN is trained by the backpropagation algorithm (2.11). Then, the closed loop of the nonlinear system is stable if the learning rates are chosen as

$$
0<\eta(k)<\frac{2}{\left(P_{\max }\right)^{2}}
$$

where $P_{\max } \equiv\left[\begin{array}{llllll}P_{1, \max } & P_{2, \max } & P_{3, \max } & P_{4, \max } & P_{5, \max } & P_{6, \max }\end{array}\right]^{T}$. In addition, One has the following optimal learning rate preserving high-speed convergence and nonlinear system stability

$$
\eta^{\mathrm{opt}}(k)=\frac{1}{\left\|Y_{u}(\partial \widehat{y}(k) / \partial W)\right\|^{2}} .
$$

Proof. See the appendix.

As above, this is a model-free control approach for nonlinear system, that is, the designed control scheme does not use the system dynamic model. In practical systems, system dynamics are not usually known exactly and the sensitivity needs to be estimated. As discussed above, the system sensitivity $Y_{u}$ can be obtained from type-2 FNNI in each iteration if the identifier is efficient and accurate (the type-2 FNN output $\hat{Y}$ can approximate the nonlinear plant output $Y$ ). Thus, $Y_{u}$ can be represented in terms of type-2 FNNI's parameters $m, \bar{\sigma}, \underline{\sigma}, w$. In general, the calculation of $Y_{u}$ needs complex computation, and it is time varying. It usually fails for real-time control problem of industrial applications. Therefore, based on the system dynamic model, a simple modification in adaptation laws is introduced below.

\section{Robust Adaptive Control Scheme Using Type-2 FNN System}

\subsection{Nonlinear System Description}

An $n$ th-order nonlinear dynamic system considered in the companion form or controllability canonical form is given by

$$
x^{(n)}=F(\mathbf{x})+G(\mathbf{x}) u+D, \quad y=x,
$$

where $u$ and $y$ are the control input and nonlinear system output, respectively. $\mathbf{x}=$

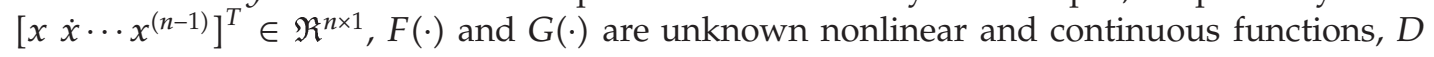
is the bounded external disturbance or system uncertainty. In order to make system (3.1) controllable, $G(\mathbf{x})$ needs to be invertible for all $\mathbf{x} \in U_{C} \subset \mathfrak{R}^{n \times 1}$. 


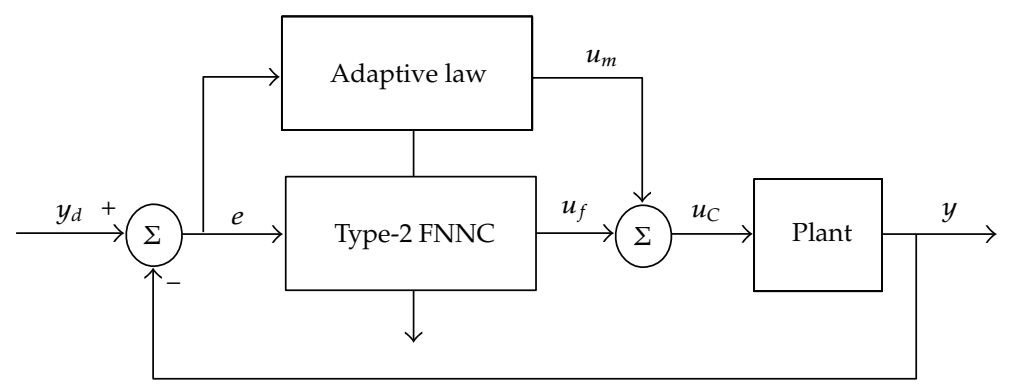

Figure 4: Robust adaptive type-2 FNN control system.

Our purpose is to design a robust adaptive control scheme which guarantees boundedness of all closed-loop variables and tracking of a given reference trajectory $\mathbf{y}_{\mathbf{d}}=$ $\left[\begin{array}{llll}y_{d} & \dot{y}_{d} \cdots & y_{d}^{(n-1)}\end{array}\right]^{T} \in \mathfrak{R}^{n}$. Define the tracking error $\mathbf{e}$ as

$$
\mathbf{e}=\mathbf{y}_{\mathbf{d}}-\mathbf{y}=\left[\begin{array}{llll}
e & \dot{e} & \cdots & e^{(n-1)}
\end{array}\right]^{T} \in \mathfrak{R}^{n} .
$$

If the plant dynamics is well known, the ideal control law $u^{*}$ can be designed by the feedback linearization approach [25]

$$
u^{*}=G(\mathbf{x})^{-1}\left[x^{(n)}-F(\mathbf{x})-D(t)+\mathbf{K e}\right]
$$

where $\mathbf{K}=\left[\begin{array}{llll}k_{n} & k_{n-1} & \cdots & k_{1}\end{array}\right] \in \mathfrak{R}^{1 \times n}$. Positive control gain $\mathbf{K}$ is chosen as $\left(k_{i}>0, I=\right.$ $1, \ldots, n)$ such that all roots of the polynomial $s^{n}+k_{1} \cdot s^{(n-1)}+\cdots+k_{n}=0$ are in the open left-half plane. Substituting (3.3) into (3.1) yields

$$
e^{(n)}+k_{1} e^{(n-1)}+\cdots+k_{n} e=0
$$

which implies that $\lim _{t \rightarrow \infty} e(t)=0$. However, the nonlinear functions $F(\mathbf{x})$ and $G(\mathbf{x})$ are not well known in general. Therefore, we cannot obtain the ideal control law (3.3). To solve this problem, the adaptive type-2 FNN control system is proposed to approximate the ideal control law (3.3).

\subsection{Design for Type-2 FNN Control System}

The configuration of the proposed robust type-2 FNN control system is depicted in Figure 4. The type-2 FNN controller $u_{f}$ is connected to the compensated controller $u_{m}$ to generate a control signal $u_{C}$. That is, the control law is given by

$$
u_{C}=u_{f}+u_{m}
$$

From (2.9), we can define the control input by type-2 FNN with uncertain variance which is used to approximate ideal control (3.3)

$$
u_{f}=\widehat{\mathbf{w}}^{T} \widehat{\mathbf{O}}_{3} .
$$


The minimum approximation error $\varepsilon$ can be defined as

$$
\varepsilon=u^{*}-u_{f}^{*}
$$

By the universal approximation theorem $[3,8,10]$, there exists optimal parameters $\mathbf{w}^{*}$ such that $u_{f}\left(\mathbf{w}^{*}\right)=u_{f}^{*}$ can approximate $u$ as close as possible. Consequently, (3.7) can be rewritten as

$$
u^{*}=u_{f}^{*}+\varepsilon=\left(\mathbf{w}^{*}\right)^{T} \mathbf{O}_{3}^{*}+\varepsilon
$$

From (3.1), (3.5), and (3.8), the system tracking error equation is rewritten as

$$
\dot{\mathbf{e}}=\Lambda \mathbf{e}+\mathbf{B}_{G}\left(u^{*}-u_{C}\right),
$$

where

$$
\Lambda=\left[\begin{array}{cccc}
0 & 1 & \cdots & 0 \\
\vdots & \vdots & \ddots & \vdots \\
0 & 0 & \cdots & 1 \\
-k_{n} & -k_{n-1} & \cdots & -k_{1}
\end{array}\right], \quad \mathbf{B}_{G}=\left[\begin{array}{c}
0 \\
\vdots \\
0 \\
G(\mathbf{x})
\end{array}\right]
$$

Subsequently, we define $\tilde{u}=u^{*}-u_{C}$, thus

$$
\begin{aligned}
\tilde{u} & =\left[u+G(\mathbf{x})^{-1} \mathbf{K e}\right]-\left(u_{f}+\mathbf{K e}+u_{m}\right) \\
& =\left[\left(\mathbf{w}^{*}\right)^{T} \mathbf{O}_{3}^{*}+\varepsilon-\widehat{\mathbf{w}}^{T} \widehat{\mathbf{O}}_{3}\right]-u_{m} \\
& =\left[\left(\mathbf{w}^{*}\right)^{T} \mathbf{O}_{3}^{*}+\varepsilon-\widehat{\mathbf{w}}^{T} \mathbf{O}_{3}^{*}+-\widehat{\mathbf{w}}^{T} \mathbf{O}_{3}^{*}-\widehat{\mathbf{w}}^{T} \widehat{\mathbf{O}}_{3}\right]-u_{m} \\
& =\left(\mathbf{w}^{*}\right)^{T} \widetilde{\mathbf{O}}_{3}+\widetilde{\mathbf{w}}^{T} \mathbf{O}_{3}^{*}+\varepsilon-u_{m} .
\end{aligned}
$$

Using the linearization technique, we have the Taylor expansion of $\widetilde{\mathbf{O}}_{3}$

$$
\begin{aligned}
& \tilde{\mathbf{O}}_{3}=\left[\begin{array}{llll}
\tilde{h}_{1} & \tilde{h}_{2} & \cdots & \tilde{h}_{l}
\end{array}\right]^{T}
\end{aligned}
$$

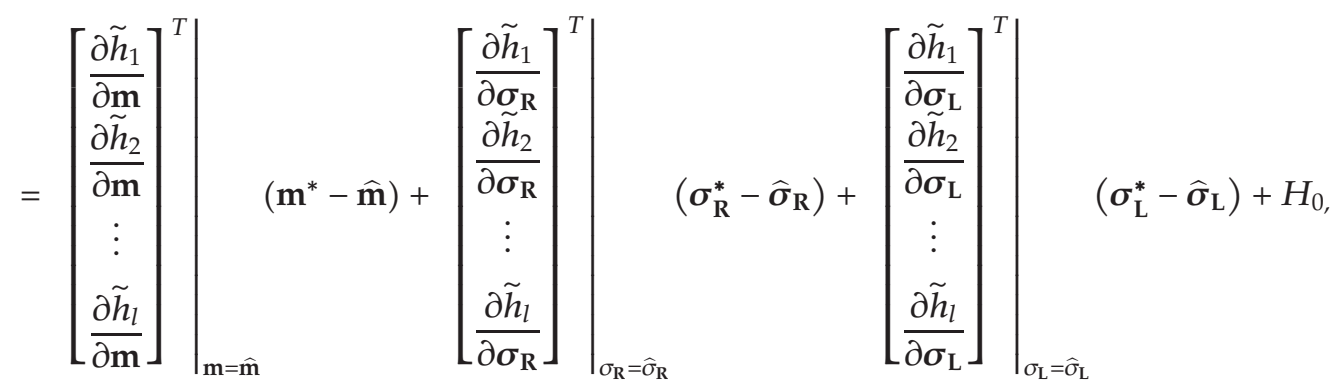


where $H_{0}$ represents higher-order terms. $\tilde{h}_{j}=\left(\bar{O}_{j}^{(3)}+\underline{O}_{j}^{(3)}\right) / 2$ and $j=1, \ldots, l$ denote the $j$ th-output of type-2 fuzzy antecedent matching. Substituting (3.12) into (3.11) gives

$$
\begin{aligned}
& \tilde{\boldsymbol{u}}=\widetilde{\mathbf{w}}^{T}\left(\widehat{\mathbf{O}}_{3}+\mathbf{O}_{\mathbf{C}}^{T} \tilde{\mathbf{m}}+\mathbf{O}_{\mathbf{R}}^{T} \widetilde{\boldsymbol{\sigma}}_{\mathbf{R}}+\mathbf{O}_{\mathbf{L}}^{T} \widetilde{\boldsymbol{\sigma}}_{\mathbf{L}}+\mathbf{H}_{0}\right)+\varepsilon+\widehat{\mathbf{w}}^{T}\left(\mathbf{O}_{\mathbf{C}}^{T} \tilde{\mathbf{m}}+\mathbf{O}_{\mathbf{R}}^{T} \widetilde{\boldsymbol{\sigma}}_{\mathbf{R}}+\mathbf{O}_{\mathbf{L}}^{T} \widetilde{\boldsymbol{\sigma}}_{\mathbf{L}}+\mathbf{H}_{0}\right)-u_{m} \\
& =\widetilde{\mathbf{w}}^{T} \widehat{\mathbf{O}}_{4}+\widehat{\mathbf{w}}^{T}\left(\mathbf{O}_{\mathbf{C}}^{T} \tilde{\mathbf{m}}+\mathbf{O}_{\mathbf{R}}^{T} \widetilde{\boldsymbol{\sigma}}_{\mathbf{R}}+\mathbf{O}_{\mathbf{L}}^{T} \widetilde{\boldsymbol{\sigma}}_{\mathbf{L}}\right)+\left(\mathbf{w}^{*}\right)^{T} \mathbf{H}_{0}+\widetilde{\mathbf{w}}^{T}\left(\mathbf{O}_{\mathbf{C}}^{T} \tilde{\mathbf{m}}+\mathbf{O}_{\mathbf{R}}^{T} \widetilde{\boldsymbol{\sigma}}_{\mathbf{R}}+\mathbf{O}_{\mathbf{L}}^{T} \widetilde{\boldsymbol{\sigma}}_{\mathbf{L}}\right)-u_{m} \\
& =\widetilde{\mathbf{w}}^{T} \widehat{\mathbf{O}}_{4}+\widehat{\mathbf{w}}^{T}\left(\mathbf{O}_{\mathbf{C}}^{T} \tilde{\mathbf{m}}+\mathbf{O}_{\mathbf{R}}^{T} \widetilde{\boldsymbol{\sigma}}_{\mathbf{R}}+\mathbf{O}_{\mathbf{L}}^{T} \tilde{\boldsymbol{\sigma}}_{\mathbf{L}}\right)-u_{m}+\Delta_{0} .
\end{aligned}
$$

From (3.9) and (3.13), we have

$$
\left.\dot{\mathbf{e}}=\Lambda \mathbf{e}+\mathbf{B}_{G} \cdot \mid \widetilde{\mathbf{w}}^{T} \widehat{\mathbf{O}}_{4}+\widehat{\mathbf{w}}^{\mathbf{T}}\left(\mathbf{O}_{\mathrm{C}}^{T} \widetilde{\mathbf{m}}+\mathbf{O}_{\mathbf{R}}^{T} \widetilde{\boldsymbol{\sigma}}_{\mathbf{R}}+\mathbf{O}_{\mathbf{L}}^{T} \widetilde{\boldsymbol{\sigma}}_{\mathbf{L}}\right)+\Delta_{0}-u_{m}\right\rfloor
$$

Theorem 3.1. Consider the nonlinear system (3.1). The adaptive control input is presented in (3.5). Thus, the adaptive control input $u_{f}$ is designed as (3.6) and compensated controller $u_{m}$ is designed as (3.16) with the estimation gain $\widehat{\delta}$ given in (3.16), where $\widehat{\mathbf{w}}=\left[\begin{array}{llll}\widehat{w}_{1} & \widehat{w}_{2} & \cdots & \widehat{w}_{l}\end{array}\right]^{T} \in \Re^{n \times 1}, \gamma_{w}, \gamma_{m}, \gamma_{R}$, $\gamma_{L}$, and $\gamma_{\delta}$ are positive constant, $\mathbf{P}$ is a symmetric positive definite matrix that satisfies

$$
\Lambda^{\mathrm{T}} \mathbf{P}+\mathbf{P} \Lambda=-\mathbf{Q}
$$

where $\mathbf{Q}$ is a symmetric positive definite matrix and is selected by the designer. As a result, the stability of the closed-loop system is guaranteed using the type-2 FNN system.

$$
\begin{gathered}
\dot{\hat{\mathbf{w}}}=\gamma_{w} \mathbf{e}^{\mathrm{T}} \mathbf{P} \mathbf{B}_{G} \widehat{\mathbf{O}}_{4}, \\
\dot{\hat{\mathbf{m}}}=\gamma_{m} \mathbf{e}^{\mathrm{T}} \mathbf{P} \mathbf{B}_{G} \mathbf{O}_{\mathbf{C}} \widehat{\mathbf{w}}, \\
\dot{\hat{\boldsymbol{\sigma}}}_{\mathbf{R}}=\gamma_{R} \mathbf{e}^{\mathrm{T}} \mathbf{P} \mathbf{B}_{G} \mathbf{O}_{\mathbf{R}} \widehat{\mathbf{w}}, \\
\dot{\hat{\boldsymbol{\sigma}}}_{\mathbf{L}}=\gamma_{L} \mathbf{e}^{\mathrm{T}} \mathbf{P} \mathbf{B}_{G} \mathbf{O}_{\mathbf{L}} \widehat{\mathbf{w}}, \\
\dot{\widehat{\delta}}=\gamma_{\delta}\left|\mathbf{e}^{\mathrm{T}} \mathbf{P} \mathbf{B}_{G}\right|, \\
u_{m}=\widehat{\delta} \operatorname{sgn}\left(\mathbf{e}^{\mathrm{T}} \mathbf{P} \mathbf{B}_{G}\right) .
\end{gathered}
$$

Proof. We consider the following Lyapunov candidate

$$
V\left(\mathbf{e}, \widetilde{\mathbf{w}}, \widetilde{\mathbf{m}}, \widetilde{\boldsymbol{\sigma}}_{\mathbf{R}}, \widetilde{\boldsymbol{\sigma}}_{\mathbf{L}}, \widetilde{\delta}, t\right)=\frac{1}{2} \mathbf{e}^{T} \mathbf{P e}+\frac{1}{2 \gamma_{w}} \widetilde{\mathbf{w}}^{T} \widetilde{\mathbf{w}}+\frac{1}{2 \gamma_{m}} \widetilde{\mathbf{m}}^{T} \widetilde{\mathbf{m}}+\frac{1}{2 \gamma_{R}} \widetilde{\boldsymbol{\sigma}}_{\mathbf{R}}^{T} \widetilde{\boldsymbol{\sigma}}_{\mathbf{R}}+\frac{1}{2 \gamma_{L}} \widetilde{\boldsymbol{\sigma}}_{\mathrm{L}}^{T} \widetilde{\boldsymbol{\sigma}}_{\mathbf{L}}+\frac{1}{2 \gamma_{\delta}} \widetilde{\delta}^{2},
$$


where the estimation error of the uncertainty bound is defined as $\widetilde{\delta}=\delta-\widehat{\delta}$. Taking the derivative of the Lyapunov candidate (3.17) yields

$$
\begin{aligned}
& \dot{V}\left(\mathbf{e}, \tilde{\mathbf{w}}, \tilde{\mathbf{m}}, \tilde{\boldsymbol{\sigma}}_{\mathbf{R}}, \tilde{\boldsymbol{\sigma}}_{\mathbf{L}}, \tilde{\boldsymbol{\delta}}, \mathbf{t}\right)=\frac{1}{2} \dot{\mathbf{e}}^{T} \mathbf{P e}+\frac{1}{2} \mathbf{e}^{T} \mathbf{P} \dot{\mathbf{e}}-\frac{1}{\gamma_{w}} \widetilde{\mathbf{w}}^{T} \dot{\tilde{\mathbf{w}}}-\frac{1}{\gamma_{m}} \tilde{\mathbf{m}}^{T} \dot{\tilde{\mathbf{m}}}-\frac{1}{\gamma_{R}} \tilde{\boldsymbol{\sigma}}_{\mathbf{R}}^{T} \dot{\tilde{\boldsymbol{\sigma}}}_{\mathbf{R}}-\frac{1}{\gamma_{L}} \tilde{\boldsymbol{\sigma}}_{\mathrm{L}}^{T} \dot{\tilde{\boldsymbol{\sigma}}}_{\mathbf{L}}-\frac{1}{\gamma_{\delta}} \tilde{\delta} \dot{\hat{\delta}} \\
& =\frac{1}{2} \mathbf{e}^{T}\left(\boldsymbol{\Lambda}^{T} \mathbf{P}+\mathbf{P} \boldsymbol{\Lambda}\right) \mathbf{e}+\frac{1}{2}\left(\mathbf{B}_{G}^{T} \mathbf{P e}+\mathbf{e}^{T} \mathbf{P} \mathbf{B}_{G}\right) \tilde{\boldsymbol{u}}-\frac{1}{\gamma_{w}} \tilde{\mathbf{w}}^{T} \dot{\tilde{\mathbf{w}}}-\frac{1}{\gamma_{m}} \tilde{\mathbf{m}}^{T} \dot{\tilde{\mathbf{m}}} \\
& -\frac{1}{\gamma_{R}} \tilde{\boldsymbol{\sigma}}_{\mathbf{R}}^{T} \dot{\widetilde{\sigma}}_{\mathbf{R}}-\frac{1}{\gamma_{L}} \tilde{\boldsymbol{\sigma}}_{\mathbf{L}}^{T} \dot{\tilde{\boldsymbol{\sigma}}}_{\mathbf{L}}-\frac{1}{\gamma_{\delta}} \tilde{\delta} \dot{\hat{\delta}} \\
& =\frac{1}{2} \mathbf{e}^{T} \mathbf{Q e}+\mathbf{e}^{T} \mathbf{P} \mathbf{B}_{G}\left[\widetilde{\mathbf{w}}^{T} \widehat{\mathbf{O}}_{4}+\widetilde{\mathbf{w}}^{\mathbf{T}} \cdot\left(\mathbf{O}_{\mathbf{m}}^{T} \tilde{\mathbf{m}}+\mathbf{O}_{\mathbf{R}}^{T} \widetilde{\boldsymbol{\sigma}}_{\mathbf{R}}+\mathbf{O}_{\mathbf{L}}^{T} \cdot \widetilde{\boldsymbol{\sigma}}_{\mathbf{L}}\right)\right] \\
& -\mathbf{e}^{T} \mathbf{P} \mathbf{B}_{G}(u m-\Delta t)-\frac{1}{\gamma_{w}} \tilde{\mathbf{w}}^{T} \dot{\tilde{\mathbf{w}}}-\frac{1}{\gamma_{m}} \tilde{\mathbf{m}}^{T} \dot{\tilde{\mathbf{m}}}-\frac{1}{\gamma_{R}} \tilde{\boldsymbol{\sigma}}_{\mathbf{R}}^{T} \dot{\tilde{\boldsymbol{\sigma}}}_{\mathbf{R}}-\frac{1}{\gamma_{L}} \tilde{\boldsymbol{\sigma}}_{\mathbf{L}}^{T} \dot{\tilde{\boldsymbol{\sigma}}}_{\mathbf{L}}-\frac{1}{\gamma_{\delta}} \tilde{\delta} \dot{\tilde{\delta}}
\end{aligned}
$$

From (3.16), (3.18) can be re-written as

$$
\begin{aligned}
\dot{V}\left(\mathbf{e}, \widetilde{\mathbf{w}}, \tilde{\mathbf{m}}, \widetilde{\boldsymbol{\sigma}}_{\mathbf{R}}, \widetilde{\boldsymbol{\sigma}}_{\mathbf{L}}, \widetilde{\delta}, t\right) & =-\frac{1}{2} \mathbf{e}^{T} \mathbf{Q} \mathbf{e}-\mathbf{e}^{T} \mathbf{P} \mathbf{B}_{G}\left(u_{m}-\Delta_{t}\right)-\frac{1}{\gamma_{\delta}} \tilde{\delta} \dot{\hat{\delta}} \\
& =-\frac{1}{2} \mathbf{e}^{T} \mathbf{Q} \mathbf{e}+\mathbf{e}^{T} \mathbf{P} \mathbf{B}_{G} \Delta_{t}-\mathbf{e}^{T} \mathbf{P} \mathbf{B}_{G} u_{m}-\frac{1}{\gamma_{\delta}} \tilde{\delta} \dot{\delta} \\
& =-\frac{1}{2} \mathbf{e}^{T} \mathbf{Q} \mathbf{e}+\mathbf{e}^{T} \mathbf{P} \mathbf{B}_{G} \Delta_{t}-\widehat{\delta}\left|\mathbf{e}^{T} \mathbf{P} \mathbf{B}_{G}\right|-(\delta-\widehat{\delta})\left|\mathbf{e}^{T} \mathbf{P} \mathbf{B}_{G}\right| \\
& \leq-\frac{1}{2} \mathbf{e}^{T} \mathbf{Q} \mathbf{e}-\left|\mathbf{e}^{T} \mathbf{P} \mathbf{B}_{G}\right|\left(\delta-\left|\Delta_{t}\right|\right) \leq 0 .
\end{aligned}
$$

As the above $\dot{V}\left(\mathbf{e}, \widetilde{\mathbf{w}}, \widetilde{\mathbf{m}}_{,} \widetilde{\boldsymbol{\sigma}}_{\mathbf{R}}, \widetilde{\boldsymbol{\sigma}}_{\mathbf{L}}, \widetilde{\delta}, t\right) \leq 0$ is a negative semidefinite function, it implies that $\mathbf{e}$, $\widetilde{\mathbf{w}}, \widetilde{\mathbf{m}}, \widetilde{\boldsymbol{\sigma}}_{\mathbf{R}}, \widetilde{\boldsymbol{\sigma}}_{\mathbf{L}}$ and $\tilde{\delta}$ are bounded. Let the function $\phi(t)=1 / 2 \mathbf{e}^{T} \mathbf{Q e} \leq-\dot{V}\left(\mathbf{e}, \widetilde{\mathbf{w}}, \widetilde{\mathbf{m}}, \widetilde{\boldsymbol{\sigma}}_{\mathbf{R}}, \widetilde{\boldsymbol{\sigma}}_{\mathbf{L}}, \widetilde{\delta}_{,} t\right)$, and integrating the function with respect to time, we have

$$
\int_{0}^{t} \phi(\tau) d \tau \leq V\left(\mathbf{e}, \widetilde{\mathbf{w}}, \tilde{\mathbf{m}}, \tilde{\boldsymbol{\sigma}}_{\mathbf{R}}, \tilde{\boldsymbol{\sigma}}_{\mathrm{L}}, \widetilde{\delta}, 0\right)-V\left(\mathbf{e}, \widetilde{\mathbf{w}}, \widetilde{\mathbf{m}}, \tilde{\boldsymbol{\sigma}}_{\mathbf{R}}, \tilde{\boldsymbol{\sigma}}_{\mathrm{L}}, \widetilde{\delta}, t\right)
$$

Since $V\left(\mathbf{e}, \widetilde{\mathbf{w}}, \widetilde{\mathbf{m}}, \widetilde{\boldsymbol{\sigma}}_{\mathrm{R}}, \widetilde{\boldsymbol{\sigma}}_{\mathrm{L}}, \widetilde{\delta}, 0\right)$ is bounded, and $V\left(\mathbf{e}, \widetilde{\mathbf{w}}, \widetilde{\mathbf{m}}, \widetilde{\boldsymbol{\sigma}}_{\mathrm{R}}, \widetilde{\boldsymbol{\sigma}}_{\mathrm{L}}, \widetilde{\delta}, t\right)$ is not increasing, that is, $V\left(\mathbf{e}, \widetilde{\mathbf{w}}, \widetilde{\mathbf{m}}, \widetilde{\boldsymbol{\sigma}}_{\mathbf{R}}, \widetilde{\sigma}_{\mathrm{L}}, \widetilde{\delta}, t\right)$ is bounded. Thus,

$$
\lim _{t \rightarrow \infty} \int_{0}^{t} \phi(\tau) d \tau \leq \infty
$$

Differentiating $\phi(t)$ with respect to time, we get

$$
\dot{\phi}(t)=\mathbf{e}^{T} \mathbf{Q} \dot{\mathbf{e}} .
$$


Since all the variables on the right side of (3.19) are bounded, which implies that $\dot{\mathbf{e}}$ is also bounded. Therefore, $\phi(t)$ is uniformly continuous [25]. By Babalat's lemma [25], it can be shown that $\lim _{t \rightarrow \infty} \phi(t)=0$. Therefore, $\lim _{t \rightarrow \infty} \mathbf{e}(t) \rightarrow 0$, the stability of the closed-loop system is guaranteed using type-2 FNN system.

Remark 3.2. Comparing the computation of control schemes shown in Figures 3 and 4, we can observe clearly that Figure 4 is less than Figure 3 (almost half) in each iteration. In addition, a compensator is designed to improve the control performance. Therefore, the adaptive control scheme shown in Figure 4 is suitable for practical applications.

\subsection{Optimal Learning Rate Algorithm for Type-2 FNN Control System}

According to the gradient method, the update laws of parameters of type-2 FNN system are shown in (2.12) and (2.13). In order to obtain the system sensitivity and reduce the computation complexity, herein, we have a comparison in (2.12) and (3.16). Rewrite (2.12) as

$$
\begin{gathered}
\Delta \widehat{w}_{j}=\eta_{w}(k) Y_{u} e\left(\frac{O_{j}^{(3)}+O_{j}^{(3)}}{2}\right), \\
\Delta \widehat{m}_{i j}=\eta_{m}(k) Y_{u} e\left[\widehat{w}_{j}\left(\frac{\partial \bar{O}_{j}^{(3)}}{\partial \hat{m}_{i j}}+\frac{\partial \underline{O}_{j}^{(3)}}{\partial \widehat{m}_{i j}}\right)\right], \\
\Delta \widehat{\sigma}_{R i j}=\eta_{R}(k) Y_{u} e\left[\widehat{w}_{j}\left(\frac{\partial \bar{O}_{j}^{(3)}}{\partial \bar{\sigma}_{i j}}+\frac{\partial \underline{O}_{j}^{(3)}}{\partial \bar{\sigma}_{i j}}\right)\right], \\
\Delta \widehat{\sigma}_{L i j}=\eta_{L}(k) Y_{u} e\left[\widehat{w}_{j}\left(\frac{\partial \bar{O}_{j}^{(3)}}{\partial \widehat{\sigma}_{i j}}+\frac{\partial \underline{O}_{j}^{(3)}}{\partial \widehat{\sigma}_{i j}}\right)\right],
\end{gathered}
$$

and then transfer (3.16) to a discrete-time form

$$
\begin{gathered}
\Delta \widehat{w}_{j} \equiv t_{s} \gamma_{w} \mathbf{e}^{\mathrm{T}} \mathbf{P} \mathbf{B}_{G} \widehat{O}_{4 j}=\rho_{w} \mathbf{e}^{\mathrm{T}} \mathbf{P} X\left(\frac{O_{j}^{(3)}+O_{j}^{(3)}}{2}\right), \\
\Delta \widehat{m}_{i j} \equiv t_{s} \rho_{m} \mathbf{e}^{\mathrm{T}} \mathbf{P} \mathbf{B}_{G} O_{C i j} \widehat{w}_{j}=\rho_{m} \mathbf{e}^{\mathrm{T}} \mathbf{P} X\left[\widehat{w}_{j}\left(\frac{\partial \bar{O}_{j}^{(3)}}{\partial \widehat{m}_{i j}}+\frac{\partial \underline{O}_{j}^{(3)}}{\partial \widehat{m}_{i j}}\right)\right], \\
\Delta \widehat{\sigma}_{R i j} \equiv t_{s} \rho_{R} \mathbf{e}^{\mathrm{T}} \mathbf{P} \mathbf{B}_{G} O_{R i j} \widehat{w}_{j}=\rho_{R} \mathbf{e}^{\mathrm{T}} \mathbf{P}_{X}\left[\widehat{w}_{j}\left(\frac{\partial \bar{O}_{j}^{(3)}}{\partial \bar{\sigma}_{i j}}+\frac{\partial \underline{O}_{j}^{(3)}}{\partial \bar{\sigma}_{i j}}\right)\right], \\
\Delta \widehat{\sigma}_{L i j} \equiv t_{s} \rho_{L} \mathbf{e}^{\mathrm{T}} \mathbf{P} \mathbf{B}_{G} O_{L i j} \widehat{w}_{j}=\rho_{L} \mathbf{e}^{\mathrm{T}} \mathbf{P} X\left[\widehat{w}_{j}\left(\frac{\partial \bar{O}_{j}^{(3)}}{\partial \widehat{\sigma}_{i j}}+\frac{\partial \underline{O}_{j}^{(3)}}{\partial \widehat{\sigma}_{i j}}\right)\right],
\end{gathered}
$$


where $t_{s}$ denotes the sampling time, $\boldsymbol{x}=\left[\begin{array}{llll}0 & \cdots & 0 & 1\end{array}\right]^{T} \in \mathfrak{R}^{n \times 1}$ and $\rho_{i}=t_{s} \gamma_{i} G(\mathbf{x}), i=$ $w, m, R, L$, can be viewed as the learning rate of parameters for the type-2 FNN system. Let $\rho_{i}=\eta_{i}, i=w, m, R, L$ and compare each equation between (3.23) and (3.24), we then have

$$
\eta_{i} Y_{u} e=\rho_{i} \mathbf{e}^{T} \mathbf{P} X, \quad i=w, m, R, L .
$$

Thus, the system sensitivity can be replaced as

$$
Y_{u}=\frac{\mathbf{e}^{T} \mathbf{P} \boldsymbol{X}}{e}
$$

Therefore, the optimal learning rate that focuses on $Y_{u}$ is chosen as

$$
\eta^{+}(k)=\frac{1}{\left\|Y_{u}(\partial \widehat{y}(k) / \partial W)\right\|^{2}} .
$$

Details about the derivation of the optimal learning rate are introduced in the appendix.

\section{Simulation Results: Tracking Control of Duffing Forced Oscillator System}

Tracking control of Duffing forced oscillator system [26, 27] is considered to illustrate the effectiveness of our approach. Consider the following Duffing forced oscillator

$$
\ddot{y}(t)+c_{2} \dot{y}(t)+c_{1} y(t)+y^{3}(t)=c_{3} \cos \left(c_{4} t\right)+u(t),
$$

where $C=\left[\begin{array}{llll}c_{1} & c_{2} & c_{3} & c_{4}\end{array}\right]$ are constant coefficients. Let $x_{1}=y(t)$ and $x_{2}=\dot{y}(t)$, system (4.1) can then be rewritten as

$$
\left[\begin{array}{l}
\dot{x}_{1} \\
\dot{x}_{2}
\end{array}\right]=\left[\begin{array}{ll}
0 & 1 \\
0 & 0
\end{array}\right]\left[\begin{array}{l}
x_{1} \\
x_{2}
\end{array}\right]+\left[\begin{array}{l}
0 \\
1
\end{array}\right](F+G u+D), \quad y=\left[\begin{array}{ll}
1 & 0
\end{array}\right]\left[\begin{array}{l}
x_{1} \\
x_{2}
\end{array}\right],
$$

where $F=-c_{1} x_{1}-c_{2} x_{2}-\left(x_{1}\right)^{3}+c_{3} \cos \left(c_{4} t\right), G=1$, and $D$ denotes the external disturbance. A square-wave is assumed with amplitude \pm 0.5 and period $2 \pi$. Here, we set $C=\left[\begin{array}{llll}1 & 0 & 12 & 1\end{array}\right]$. The sampling time is chosen as 0.01 second, and the initial state value, $\mathbf{x}(0)=\left[\begin{array}{ll}3 & 3\end{array}\right]^{T}$. The simulation results of Duffing forced oscillator are shown in Figure 5. The oscillation phenomenon is found. Our control objective is to use the adaptive type-2 FNN control scheme such that the output to track the desired trajectory. Herein, the following four cases are considered to have comparisons.

Case 1: type-2 FNNC with uncertain variance using $\eta^{+}$(optimal learning rate) and $\eta_{\text {fixed }}$.

Case 2: type-2 FNNC with uncertain mean using $\eta^{+}$(optimal learning rate) and $\eta_{\text {fixed }}$.

Case 3: type-1 and type-2 FNNC with uncertain variance using the optimal learning rate $\eta^{+}$.

Case 4: type-1 and type-2 FNNC with uncertain mean using the optimal learning rate $\eta^{+}$. 


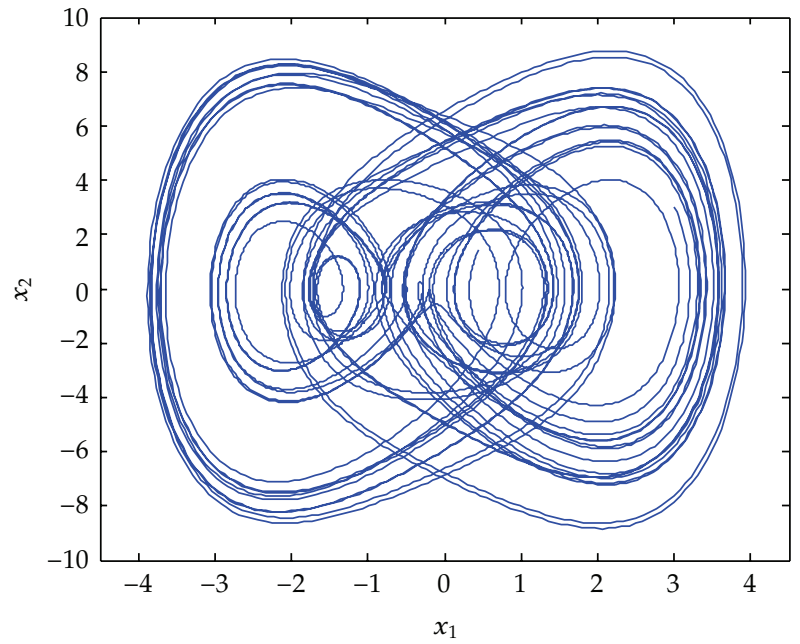

Figure 5: The phase-plant trajectory of Duffing forced oscillator without controlled.

Case 1. Type-2 FNNC with uncertain variance using $\eta^{+}$(optimal learning rate) and $\eta_{\text {fixed }}$ to compare the simulation results with different learning rates, we construct the controller using type-2 FNN system with uncertain variance. First, the parameters are chosen as

$$
\mathbf{K}=\left[\begin{array}{ll}
4 & 4
\end{array}\right]^{T}, \quad \mathbf{Q}=\left[\begin{array}{cc}
20 & 4 \\
4 & 3
\end{array}\right], \quad \mathbf{P}=\left[\begin{array}{cc}
10 & 2.5 \\
2.5 & 1
\end{array}\right]
$$

The number of rules of type-2 FNN with uncertain variance is set to be eight, and the initial values of the coefficients are chosen as

$$
\begin{aligned}
& \mathbf{x}(0)=\left[\begin{array}{ll}
3 & 3
\end{array}\right]^{T}, \quad m_{i}=\left[\begin{array}{llllllll}
-\frac{5}{2} & -\frac{25}{14} & -\frac{15}{14} & \frac{5}{14} & \frac{5}{14} & \frac{15}{14} & \frac{25}{14} & \frac{5}{2}
\end{array}\right], \\
& \sigma_{i j}=\frac{10}{7}, \quad \bar{\sigma}_{i j}=\frac{15}{7}, \quad \underline{\sigma}_{i j}=\frac{5}{7}, \quad w_{j}=0, \quad \delta(0)=0.01, \quad \eta_{\delta}=0.01 .
\end{aligned}
$$

The fixed learning rate $\rho_{i}, i=w, m, R, L$ is redefined as

$$
\eta_{\text {fixed }}=\left\{\begin{array}{l}
\eta_{m s} \equiv \rho_{i}=0.1, \quad i=m, R, L, \\
\eta_{w} \equiv \rho_{w}=1 .
\end{array}\right.
$$

Note that the optimal learning rate will be invalid when the initial weight is $w_{j}=0$. According to the literature in [7], the learning rate is usually chosen as $10^{-3} \leq \eta \leq 10$. Thus, we 


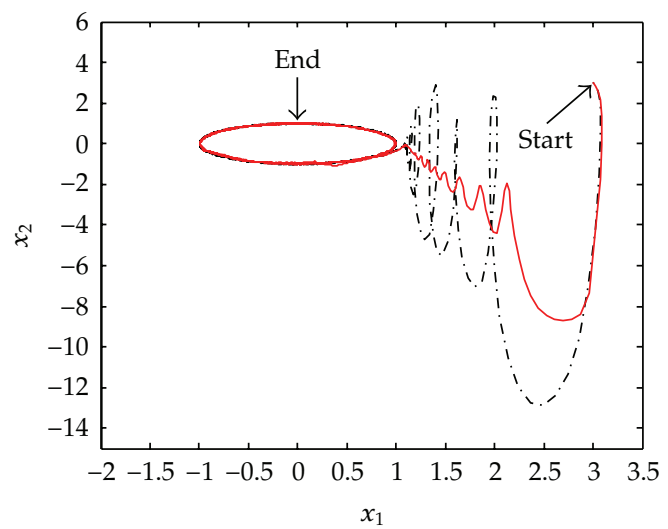

-. - Phase plant using type-2 FNNC with $\eta_{\text {fixed }}$

- Phase plant using type-2 FNNC with $\eta^{+}$

(a)

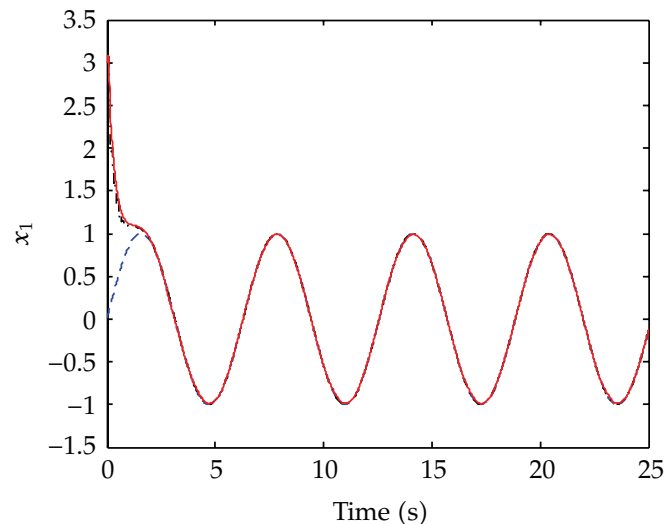

- - - Reference trajectory $y_{d}$

. . . $x_{1}$ using type-2 FNNC with $\eta_{\text {fixed }}$

- $x_{1}$ using type-2 FNNC with $\eta^{+}$

(b)

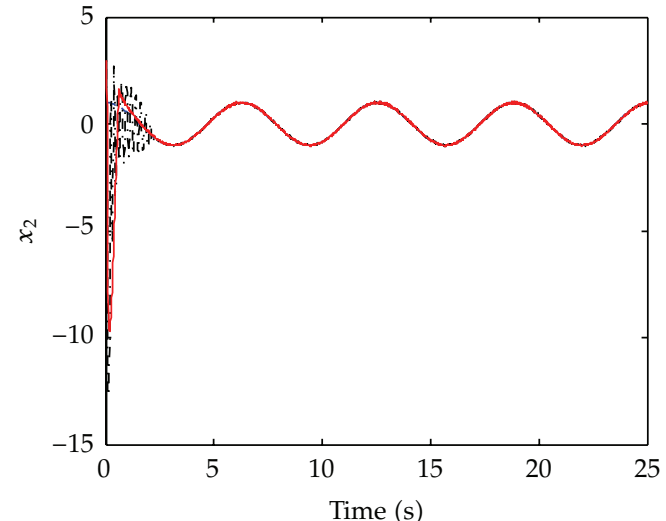

- - - Reference trajectory $y_{d}$

- . $x_{2}$ using type-2 FNNC with $\eta_{\text {fixed }}$

- $x_{2}$ using type-2 FNNC with $\eta^{+}$

(c)

Figure 6: Comparison results of Case 1 for example, (a) phase plant trajectory $\left(x_{1}, x_{2}\right)$; (b) state $x_{1}$ and its reference trajectory $y_{d} ;(\mathrm{c})$ state $x_{2}$ and its reference trajectory $\dot{y}_{d}$.

have the optimal learning rate. Hence, the optimal learning rate $\eta^{+}$of type- $2 \mathrm{FNN}$ is defined as

$$
\begin{aligned}
\eta^{+} & =\left[\begin{array}{llll}
\eta_{w_{j}}^{+} & \eta_{m_{i j}}^{+} & \eta_{\bar{\sigma}_{i j}}^{+} & \eta_{\underline{\sigma}_{i j}}^{+}
\end{array}\right] \\
& \equiv\left[\min \left(5, \frac{\partial E_{c}}{\partial w_{j}}\right) \min \left(1, \frac{\partial E_{c}}{\partial m_{i j}}\right) \min \left(1, \frac{\partial E_{c}}{\partial \bar{\sigma}_{i j}}\right) \min \left(1, \frac{\partial E_{c}}{\partial \underline{\sigma}_{i j}}\right)\right]
\end{aligned}
$$

The simulation results are shown in Figure 6. 
Case 2. Type-2 FNNC with uncertain mean using $\eta^{+}$(optimal learning rate) and $\eta_{\text {fixed }}$; in this case, type-2 FNN system with uncertain mean is considered. First, the parameters, $\mathbf{K}, \mathbf{Q}, \mathbf{P}$, are chosen as (4.3). The rule number of type-2 FNN with uncertain mean is set to be eight, and the initial values of the coefficients is chosen as

$$
\begin{aligned}
& \mathbf{x}(0)=\left[\begin{array}{ll}
3 & 3
\end{array}\right]^{T}, \quad m_{i}=\left[\begin{array}{llllllll}
-8 & -\frac{40}{7} & -\frac{24}{7} & -\frac{8}{7} & \frac{8}{7} & \frac{24}{7} & \frac{40}{7} & 8
\end{array}\right], \\
& \sigma_{i j}=\frac{16}{7}, \quad \bar{w}_{j}=\underline{w}_{j}=0, \quad \delta(0)=0.01, \quad \eta_{\delta}=0.01 .
\end{aligned}
$$

The fixed learning rate $\rho_{i}, i=w, m, R, L$ is re-defined as

$$
\eta_{\text {fixed }}=\left\{\begin{array}{l}
\eta_{m s} \equiv \rho_{i}=1, \quad i=m, R, L, \\
\eta_{w} \equiv \rho_{w}=5 .
\end{array}\right.
$$

As the above discussion, the optimal learning rate $\eta^{+}$of type-2 FNN with uncertain mean is

$$
\begin{aligned}
& \eta^{+}=\left[\begin{array}{lllll}
\eta_{\bar{w}_{j}}^{+} & \eta_{\underline{w}_{j}}^{+} & \eta_{\bar{m}_{i j}}^{+} & \eta_{\underline{m}_{i j}}^{+} & \eta_{\sigma_{i j}}^{+}
\end{array}\right] \\
& \equiv\left[\min \left(5, \frac{\partial E_{c}}{\partial \bar{w}_{j}}\right) \min \left(5, \frac{\partial E_{c}}{\partial \underline{w}_{j}}\right) \min \left(1, \frac{\partial E_{c}}{\partial \bar{m}_{i j}}\right) \min \left(1, \frac{\partial E_{c}}{\partial \underline{m}_{i j}}\right) \min \left(1, \frac{\partial E_{c}}{\partial \sigma_{i j}}\right)\right] \text {. }
\end{aligned}
$$

Simulation results of Case 2 are shown in Figure 7.

From Figures 6 and 7, we observe that the proposed robust adaptive controller with appropriate design parameters can achieve tracking control and good performance. The oscillation with large magnitude phenomenon (state $x_{2}$ ) was found in Figures 6(c) and 7(c) if the learning rates of type-2 FNNC are fixed. On the other hand, the optimal learning has better transient and steady performance compared with the simulation results using fixed learning rates.

Case 3. Type- 1 and type-2 FNNC with uncertain variance using $\eta^{+}$; to compare the simulation results with different controllers, we construct the controllers using type-2 FNN and type-1 FNN systems. First, the parameters, K, Q, P, are chosen as (4.3). The number of rules for both FNN systems are set to be eight, and the initial values of the coefficients are chosen as in Case 1. The optimal learning rate $\eta^{+}$of type- 1 FNN is defined as

$$
\eta^{+}=\left[\begin{array}{lll}
\eta_{w_{j}}^{+} & \eta_{m_{i j}}^{+} & \eta_{\sigma_{i j}}^{+}
\end{array}\right] \equiv\left[\min \left(5, \frac{\partial E_{c}}{\partial w_{j}}\right) \min \left(1, \frac{\partial E_{c}}{\partial m_{i j}}\right) \min \left(1, \frac{\partial E_{c}}{\partial \sigma_{i j}}\right)\right] .
$$

The simulation results and comparison are shown in Figure 8.

Case 4. Type-1 and type-2 FNNC with uncertain mean using the optimal learning rate $\eta^{+}$; to compare the simulation result with different controllers, we construct the controllers using type-1 FNN and type-2 FNN with uncertain mean. Firstly, the parameters, K, Q, P, are chosen 


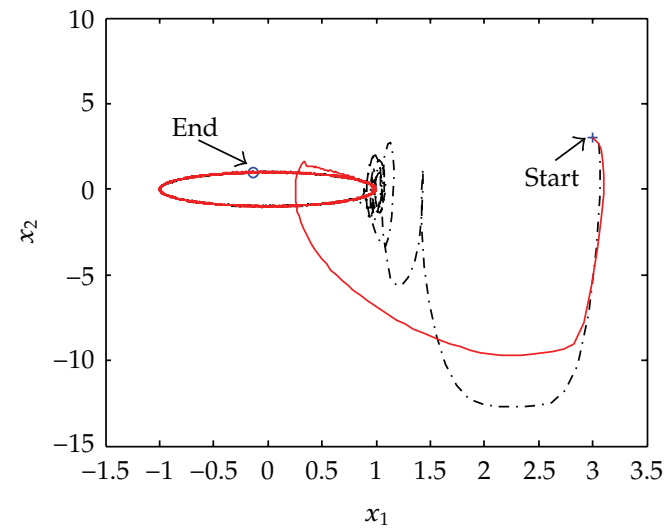

-. - Phase plant trajectory using type-2 FNNC with $\eta_{\text {fixed }}$

- Phase plant trajectory using type-2 FNNC with $\eta^{+}$

(a)

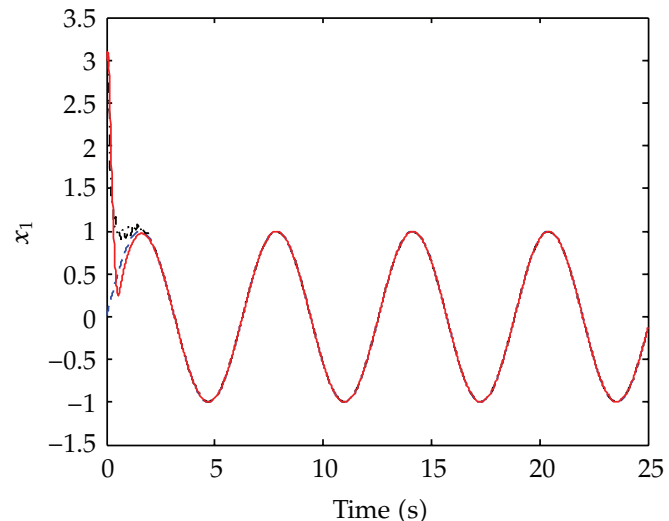

- - Reference trajectory $y_{d}$

-. - $x_{1}$ using type-2 FNNC with $\eta_{\text {fixed }}$

- $x_{1}$ using type-2 FNNC with $\eta^{+}$

(b)

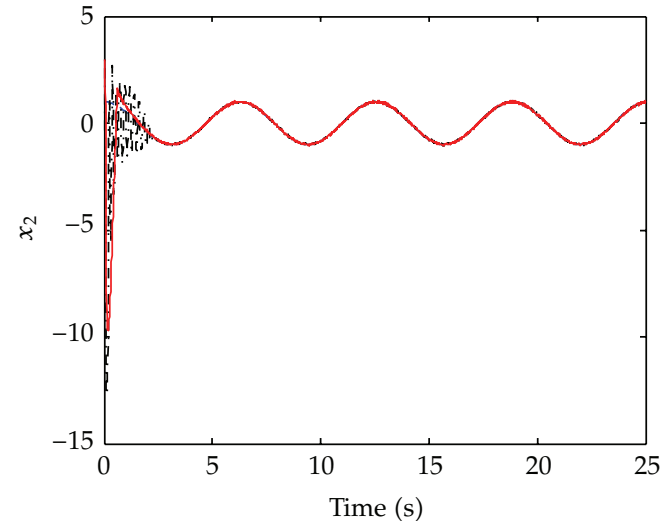

- - - Reference trajectory $y_{d}$

... $x_{2}$ using type-2 FNNC with $\eta_{\text {fixed }}$

- $x_{2}$ using type-2 FNNC with $\eta^{+}$

(c)

Figure 7: Comparison results of Case 2 for example, (a) phase plant trajectory $\left(x_{1}, x_{2}\right)$; (b) state $x_{1}$ and its reference trajectory $y_{d} ;(\mathrm{c})$ state $x_{2}$ and its reference trajectory $\dot{y}_{d}$.

as (4.3).The rule number of both FNN systems are set to be eight, and the initial values of the coefficients are chosen as

$$
\begin{aligned}
& \mathbf{x}(0)=\left[\begin{array}{ll}
3 & 3
\end{array}\right]^{T}, \quad m_{i}=\left[\begin{array}{llllllll}
-8 & -\frac{40}{7} & -\frac{24}{7} & -\frac{8}{7} & \frac{8}{7} & \frac{24}{7} & \frac{40}{7} & 8
\end{array}\right], \\
& \sigma_{i j}=\frac{32}{7}, \quad \bar{w}_{j}=\underline{w}_{j}=0, \quad \delta(0)=0.01, \quad \eta_{\delta}=0.01 .
\end{aligned}
$$

The optimal learning rate $\eta^{+}$of type-1 FNN is defined as (4.10). Simulation results of Case 4 are shown in Figure 9. 


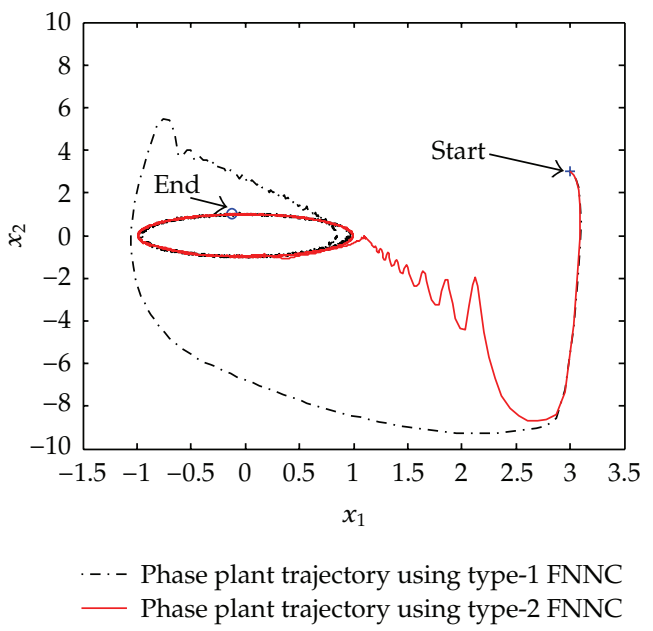

(a)

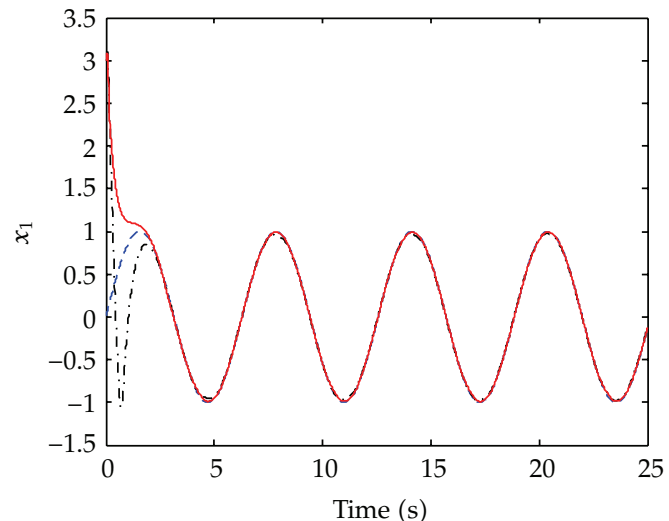

- - - Reference trajectory $y_{d}$

... $x_{1}$ using type-1 FNNC

- $x_{1}$ using type-2 FNNC

(b)

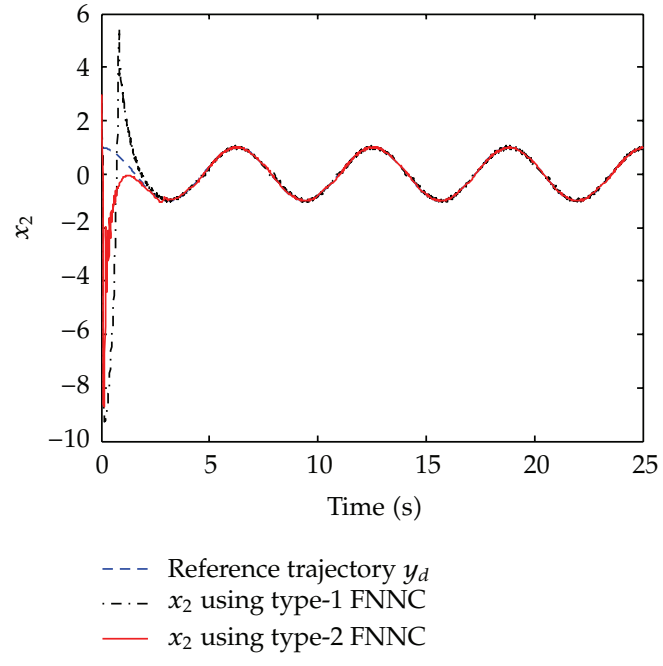

(c)

Figure 8: Comparison results of Case 3: (a) phase plant trajectory $\left(x_{1}, x_{2}\right)$; (b) state $x_{1}$ and its reference trajectory $y_{d} ;(\mathrm{c})$ state $x_{2}$ and its reference trajectory $\dot{y}_{d}$.

From Figures 8 and 9, we also find type-2 FNNCs have small convergent time, tracking error, and transient response. Besides, the type-1 FNNC has undershoot phenomenon and oscillation results in Figures 8(b), 8(c), and 9(c).

In addition, comparison results in parameters number are shown in Table 1. Rule numbers 2, 4, 6, 8 are chosen to obtain the comparison. The type-1 FNNC with 2 rules has unstable result and type-2 FNNC achieves the tracking problem. In addition, it can be observed that the tracking performance can be improved by increasing the adjustable parameters number for both type-2 and type-1 FNNCs. Besides, type-2 FNNC has smaller rules number to achieve desired specified tracking error due to more adjustable parameters. From Figures 6 and 7 and Table 2, the control performance of type-2 FNNC with uncertain variance is better than the results of type-2 FNNC with uncertain mean even it has smaller parameters. 


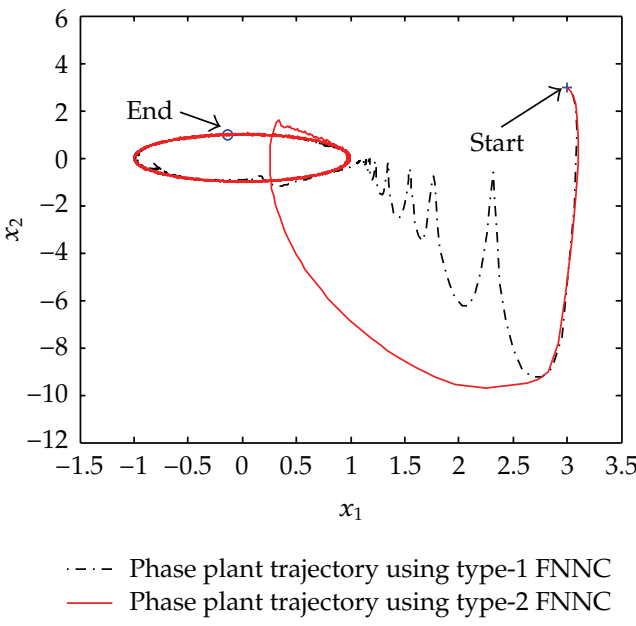

(a)

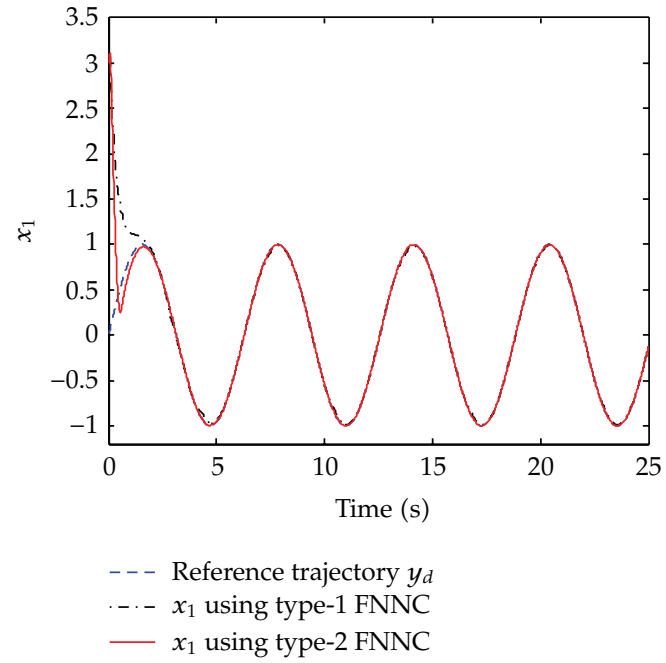

(b)

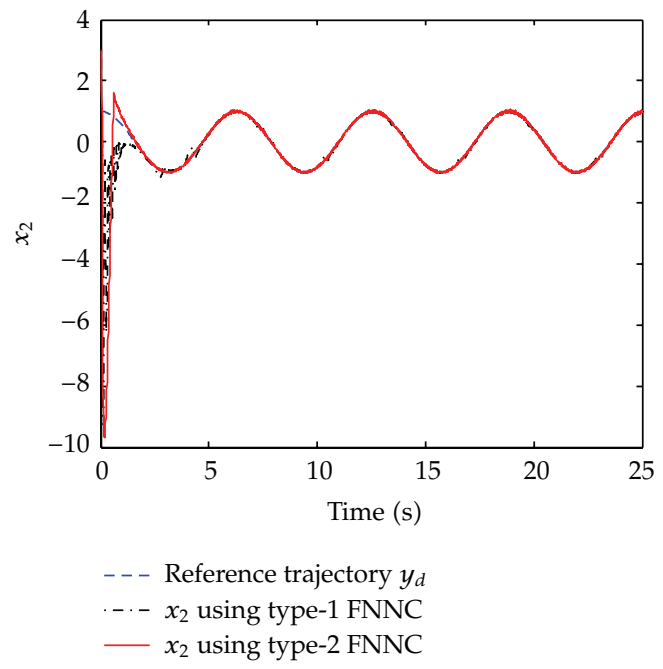

(c)

Figure 9: Comparison results of Case 4: (a) phase plant trajectory $\left(x_{1}, x_{2}\right)$; (b) state $x_{1}$ and its reference trajectory $y_{d} ;(\mathrm{c})$ state $x_{2}$ and its reference trajectory $\dot{y}_{d}$.

Finally, a comparison results in magnitude of compensator are shown in Table 2. This shows the chattering magnitude of compensated controller in steady state. Since the compensator is used to cover the approximation error of type-2 FNNC, the corresponding control effort is proportional to the approximation error. Therefore, we can conclude that the control magnitude of compensator is inversely to the rule number.

The control performance of the proposed adaptive control scheme has been demonstrated in the above simulations. For a type-2 FNN controller within an optimal and fixed learning rate, we can easily find the difference in control input of the simulations. The type-2 FNN with an optimal learning rate has better performance. In addition, within type-2 and type-1 FNN controllers, the same phenomenon exists. 
Table 1: comparison results in parameters number and tracking error.

\begin{tabular}{lcccccc}
\hline Rule number $R$ & $\begin{array}{c}\text { Type-2 FNN with } \\
\text { uncertain mean } \\
\text { Parameter } \\
\text { no. }\end{array}$ & MSE $(e)$ & $\begin{array}{c}\text { Type-2 FNN with } \\
\text { uncertain variance } \\
\text { Parameter } \\
\text { no. }\end{array}$ & MSE $(e)$ & $\begin{array}{c}\text { Parameter } \\
\text { no. }\end{array}$ & MSE (e) \\
\hline 2 & 16 & 0.262 & 14 & 0.249 & 10 & Diverge \\
4 & 32 & 0.2417 & 28 & 0.235 & 20 & 0.258 \\
6 & 48 & 0.23 & 42 & 0.159 & 30 & 0.26 \\
8 & 64 & 0.114 & 56 & 0.088 & 40 & 0.245 \\
\hline
\end{tabular}

Table 2: Comparison results in magnitude of compensated controller.

\begin{tabular}{lccc}
\hline Rule number $R$ & Type-2 FNN with uncertain mean & Type-2 FNN with uncertain variance & Type-1 FNN \\
\hline 4 & 24 & 12 & 56 \\
6 & 6 & 8 & 18 \\
\hline
\end{tabular}

\section{Conclusions}

This paper has presented a type-2 FNN system and the corresponding optimal learning algorithm for its applications. Therefore, the previous results of the type- 1 FNN have been extended to a type- 2 one. Then, the adaptive type- 2 FNN is employed to achieve the desired control performance. In the adaptive control input, the type-2 FNN controller is utilized to mimic an ideal control law with the optimal learning rate derived by the suitable substitute for system sensitivity, and the compensated controller is designed to recover the residual part of the approximation error. The closed-loop system stability has been guaranteed by the adaptive laws derived according to the Lyapunov theory. The optimal learning rate for better parameter convergence has also been guaranteed by the suitable substitute for system sensitivity. The simulation results have been presented to show the effectiveness of our approach.

\section{Appendix}

Proof of Theorem 2.1. Define the Lyapunov candidate function as in (2.10), that is, $V(k)=$ $E(k)=E_{C}$, thus we have

$$
\Delta V(k)=E(k+1)-E(k)=\frac{1}{2}\left[e^{2}(k+1)-e^{2}(k)\right]=\frac{1}{2} \cdot \Delta e(k)[2 e(k)+\Delta e(k)] .
$$

From (2.12) and (A.1)

$$
\Delta e(k)=\frac{\partial e(k)}{\partial W} \Delta W=\frac{\partial[R(k)-Y(k)]}{\partial W}[-\eta(k)] \frac{\partial E(k)}{\partial W}=\eta(k) Y_{u} \frac{\partial \widehat{y}(k)}{\partial W} \frac{\partial E(k)}{\partial W}
$$


then

$$
\frac{\partial E(k)}{\partial W}=\frac{\partial}{\partial W}\left[\frac{1}{2} e^{2}(k)\right]=e(k) \frac{\partial e(k)}{\partial W}=-e(k) Y_{u} \frac{\partial \widehat{y}(k)}{\partial W} .
$$

Therefore, (A.2) can be rewritten as

$$
\Delta e(k)=-\eta(k)\left[Y_{u} \frac{\partial \widehat{y}(k)}{\partial W}\right]^{2} e(k)
$$

Then, (A.4) can be substituted into (A.1), that is,

$$
\begin{aligned}
\Delta V(k) & =\frac{1}{2}\left[-\eta(k)\left(Y_{u} \frac{\partial \widehat{y}(k)}{\partial W}\right)^{2} e(k)\right] \cdot\left[2 e(k)-\eta(k)\left(Y_{u} \frac{\partial \widehat{y}(k)}{\partial W}\right)^{2} e(k)\right] \\
& =\frac{1}{2} \eta(k) e^{2}(k)\left(Y_{u} \frac{\partial \widehat{y}(k)}{\partial W}\right)^{2}\left[(-2+\eta k)\left(Y_{u} \frac{\partial \widehat{y}(k)}{\partial W}\right)^{2}\right] .
\end{aligned}
$$

If the following inequality holds, then $\Delta V(k) \leq 0$ will be held.

$$
0<\eta(k)<2\left[Y_{u} \frac{\partial \widehat{y}(k)}{\partial W}\right]^{-2}
$$

since

$$
\begin{aligned}
& P_{\text {max }} \equiv\left[\begin{array}{llllll}
P_{1, \max } & P_{2, \max } & P_{3, \max } & P_{4, \max } & P_{5, \max } & P_{6, \max }
\end{array}\right]^{T} \\
& =\left[\max _{i j}\left|Y_{u} \frac{\partial \widehat{y}(k)}{\partial \bar{m}_{i j}}\right| \max _{i j}\left|Y_{u} \frac{\partial \widehat{y}(k)}{\partial \underline{m}_{i j}}\right| \max _{i j}\left|Y_{u} \frac{\partial \widehat{y}(k)}{\partial \bar{\sigma}_{i j}}\right|\right. \\
& \left.\max _{i j}\left|Y_{u} \frac{\partial \widehat{y}(k)}{\partial \underline{\sigma}_{i j}}\right| \max _{i j}\left|Y_{u} \frac{\partial \widehat{y}(k)}{\partial \bar{w}_{i j}}\right| \max _{i j}\left|Y_{u} \frac{\partial \widehat{y}(k)}{\partial \underline{w}_{i j}}\right|\right] .
\end{aligned}
$$

Note that we use Gaussian MFs with uncertain variance (as Figure 2(b)) to build the membership layer, thus $\bar{m}_{i j}=\underline{m}_{i j}$ and $\bar{w}_{i j}=\underline{w}_{i j}$. Therefore, (A.7) can be simplified as

$$
\begin{aligned}
P_{\max } & \equiv\left[\begin{array}{llll}
P_{m} & P_{2} & P_{3} & P_{w}
\end{array}\right]^{T} \\
& =\left[\max _{i j}\left|Y_{u} \frac{\partial \widehat{y}(k)}{\partial m_{i j}}\right| \max _{i j}\left|Y_{u} \frac{\partial \widehat{y}(k)}{\partial \bar{\sigma}_{i j}}\right| \max _{i j}\left|Y_{u} \frac{\partial \widehat{y}(k)}{\partial \underline{\sigma}_{i j}}\right| \max _{i j}\left|Y_{u} \frac{\partial \widehat{y}(k)}{\partial w_{i j}}\right|\right]^{T}
\end{aligned}
$$


where

$$
\begin{aligned}
P_{m} & =Y_{u} \frac{\partial \widehat{y}(k)}{\partial m_{i j}}=\frac{1}{2} Y_{u} \sum_{j=1}^{l}\left(\frac{\partial \bar{O}_{j}^{(3)}}{\partial m_{i j}}+\frac{\partial \underline{O}_{j}^{(3)}}{\partial m_{i j}}\right) w_{j}^{(4)} \\
& =\frac{1}{2} Y_{u} \sum_{j=1}^{l}\left(\bar{O}_{j}^{(3)} \frac{2\left(x_{i}-m_{i j}\right)}{\left(\bar{\sigma}_{i j}\right)^{2}}+\underline{O}_{j}^{(3)} \frac{2\left(x_{i}-m_{i j}\right)}{\left(\underline{\sigma}_{i j}\right)^{2}}\right) w_{j}^{(4)} \\
& \leq \frac{1}{2} Y_{u} \sum_{j=1}^{l}\left\{\max \left[\frac{2\left(x_{i}-m_{i j}\right)}{\left(\bar{\sigma}_{i j}\right)^{2}}\right]+\max \left[\frac{2\left(x_{i}-m_{i j}\right)}{\left(\underline{\sigma}_{i j}\right)^{2}}\right]\right\} w_{j}^{(4)}, \\
P_{2} & =Y_{u} \frac{\partial \widehat{y}(k)}{\partial \bar{\sigma}_{i j}}=\frac{1}{2} Y_{u} \sum_{j=1}^{l}\left(\frac{\partial \bar{O}_{j}^{(3)}}{\partial \bar{\sigma}_{i j}}+\frac{\partial \underline{O}_{j}^{(3)}}{\partial \bar{\sigma}_{i j}}\right) w_{j}^{(4)}=\frac{1}{2} Y_{u} \sum_{j=1}^{l}\left[\bar{O}_{j}^{(3)} \frac{\left(x_{i}-m_{i j}\right)^{2}}{\left(\bar{\sigma}_{i j}\right)^{3}}\right] w_{j}^{(4)}, \\
P_{3} & =Y_{u} \frac{\partial \widehat{y}(k)}{\partial \underline{\sigma}_{i j}}=\frac{1}{2} Y_{u} \sum_{j=1}^{l}\left(\frac{\partial \bar{O}_{j}^{(3)}}{\partial \underline{\sigma}_{i j}}+\frac{\partial \underline{O}_{j}^{(3)}}{\partial \underline{\sigma}_{i j}}\right) w_{j}^{(4)}=\frac{1}{2} Y_{u} \sum_{j=1}^{l}\left[\underline{O}_{j}^{(3)} \frac{\left(x_{i}-m_{i j}\right)^{2}}{\left(\underline{\sigma}_{i j}\right)^{3}}\right] w_{j}^{(4)}, \\
P_{w} & =Y_{u} \frac{\partial \widehat{y}(k)}{\partial w_{j}^{(4)}}=\frac{1}{2} Y_{u} \sum_{j=1}^{l}\left(\bar{O}_{j}^{(3)}+\underline{O}_{j}^{(3)}\right) .
\end{aligned}
$$

In addition, the error difference can be re-expressed as

$$
e(k+1)=e(k)+\Delta e(k) \approx e(k)-\eta_{W}(k) e(k)\left(Y_{u} \frac{\partial \widehat{y}}{\partial W}\right)^{2} .
$$

Then,

$$
|e(k+1)|=\left|e(k)-\eta_{W}(k) e(k)\left(Y_{u} \frac{\partial \hat{y}}{\partial W}\right)^{2}\right| \leq|e(k)|\left|1-\eta_{W}\left(Y_{u} \frac{\partial \widehat{y}}{\partial W}\right)^{2}\right| .
$$

Thus, the so-called optimal learning rate is obtained as [2-4]

$$
\eta_{W}^{+}=\left(Y_{u} \frac{\partial \widehat{y}}{\partial W}\right)^{-2}
$$


The detail of update laws for optimal learning rate by type-2 FNN with uncertain variance are

$$
\begin{aligned}
& \eta_{\left.w_{j}^{+}\right)}^{+}=\left(Y_{u} \frac{\partial \hat{y}}{\partial w_{j}^{(4)}}\right)^{-2}=\left(Y_{u}\right)^{-2}\left(\frac{\underline{O}_{j}^{(3)}+\bar{O}_{j}^{(3)}}{2}\right)^{-2}, \\
& \eta_{m_{i j}}^{+}=\left(Y_{u} \frac{\partial \hat{y}}{\partial m_{i j}}\right)^{-2}=\left(Y_{u}\right)^{-2}\left(\frac{\partial \widehat{y}}{\partial \bar{O}_{j}^{(3)}} \frac{\partial \bar{O}_{j}^{(3)}}{\partial m_{i j}}+\frac{\partial \widehat{y}}{\partial \underline{O}_{j}^{(3)}} \frac{\partial \underline{O}_{j}^{(3)}}{\partial m_{i j}}\right)^{-2} \\
& =\left(Y_{u}\right)^{-2}\left[\frac{w_{j}^{(4)}}{2} \cdot\left(\bar{O}_{j}^{(3)} \frac{x_{i}-m_{i j}}{\bar{\sigma}_{i j}{ }^{2}}+\underline{O}_{j}^{(3)} \frac{x_{i}-m_{i j}}{\underline{\sigma}_{i j}{ }^{2}}\right)\right]^{-2}, \\
& \eta_{\bar{\sigma}_{i j}}^{+}=\left(Y_{u}^{\mathrm{opt}} \frac{\partial \widehat{y}}{\partial \bar{\sigma}_{i j}}\right)^{-2}=\left(Y_{u}^{\mathrm{opt}}\right)^{-2}\left(\frac{\partial \hat{y}}{\partial \bar{O}_{j}^{(3)}} \frac{\partial \bar{O}_{j}^{(3)}}{\partial \bar{\sigma}_{i j}}+\frac{\partial \widehat{y}}{\partial \underline{O}_{j}^{(3)}} \frac{\partial \underline{O}_{j}^{(3)}}{\partial \bar{\sigma}_{i j}}\right)^{-2} \\
& =\left(Y_{u}^{\mathrm{opt}}\right)^{-2}\left[\frac{w_{j}^{(4)}}{2} \bar{O}_{j}^{(3)} \frac{\left(x_{i}-m_{i j}\right)^{2}}{\bar{\sigma}_{i j}{ }^{3}}\right]^{-2}, \\
& \eta_{\underline{q}_{i j}}^{+}=\left(Y_{u} \frac{\partial \widehat{y}}{\partial \underline{\sigma}_{i j}}\right)^{-2}=\left(Y_{u}\right)^{-2}\left(\frac{\partial \widehat{y}}{\partial \bar{O}_{j}^{(3)}} \frac{\partial \bar{O}_{j}^{(3)}}{\partial \underline{\sigma}_{i j}}+\frac{\partial \widehat{y}}{\partial \underline{O}_{j}^{(3)}} \frac{\partial \underline{O}_{j}^{(3)}}{\partial \underline{\sigma}_{i j}}\right)^{-2} \\
& =\left(Y_{u}\right)^{-2}\left[\frac{w_{j}^{(4)}}{2} \underline{O}_{j}^{(3)} \frac{\left(x_{i}-m_{i j}\right)^{2}}{\underline{\sigma}_{i j}{ }^{3}}\right]^{-2} \text {. }
\end{aligned}
$$

By the same way, we also get the detail of update law for optimal learning rate by type-2 FNN with uncertain mean being

$$
\begin{aligned}
\eta_{w_{R j}^{(4)}}^{+} & =\left(Y_{u} \frac{\partial \hat{y}}{\partial w_{R j}^{(4)}}\right)^{-2}=\left(Y_{u} \frac{\bar{O}_{j}^{(3)}}{2}\right)^{-2}, \\
\eta_{w_{L j}(4)}^{+} & =\left(Y_{u} \frac{\partial \widehat{y}}{\partial w_{L j}^{(4)}}\right)^{-2}=\left(Y_{u} \frac{O_{j}^{(3)}}{2}\right)^{-2}, \\
\eta_{\bar{m}_{i j}^{+}} & =\left(Y_{u} \frac{\partial \hat{y}}{\partial \bar{m}_{i j}}\right)^{-2}=\left(Y_{u}\right)^{-2}\left(\frac{\partial \widehat{y}}{\partial \bar{O}_{j}^{(3)}} \frac{\partial \bar{O}_{j}^{(3)}}{\partial \bar{m}_{i j}}+\frac{\partial \widehat{y}}{\partial \underline{O}_{j}^{(3)}} \frac{\partial \underline{O}_{j}^{(3)}}{\partial \bar{m}_{i j}}\right)^{-2} \\
& =\left(Y_{u}\right)^{-2}\left[\frac{w_{R j}^{(4)}}{2} \cdot\left(\bar{O}_{j}^{(3)} \frac{x_{i}-\bar{m}_{i j}}{\sigma_{i j}^{2}}\right)\right]^{-2}, \\
\eta_{\underline{m}}^{+} & =\left(Y_{u} \frac{\partial \widehat{y}}{\partial \underline{m}_{i j}}\right)^{-2}=\left(Y_{u}\right)^{-2}\left(\frac{\partial \widehat{y}}{\partial \bar{O}_{j}^{(3)}} \frac{\partial \bar{O}_{j}^{(3)}}{\partial \underline{m}_{i j}}+\frac{\partial \widehat{y}}{\partial \underline{O}_{j}^{(3)}} \frac{\partial \underline{O}_{j}^{(3)}}{\partial \underline{m}_{i j}}\right)^{-2} \\
& =\left(Y_{u}\right)^{-2}\left[\frac{w_{L j}^{(4)}}{2} \cdot\left(\bar{O}_{j}^{(3)} \frac{x_{i}-\underline{m}_{i j}}{\sigma_{i j}^{2}}\right)\right]^{-2} \cdot
\end{aligned}
$$

This completes the proof. 


\section{Acknowledgments}

The authors would like to thank the associate editor and anonymous reviewers for their valuable comments and helpful suggestions. This work was supported by the National Science Council, Taiwan, under Contract NSC-97-2221-E-155-033-MY3.

\section{References}

[1] O. Castillo and P. Melin, "Adaptive noise cancellation using type-2 fuzzy logic and neural networks," in Proceedings of the IEEE International Conference on Fuzzy Systems (FUZZ '04), pp. 1093-1098, Budapest, Hungary, July 2004.

[2] Y. C. Chen and C. C. Teng, "A model reference control structure using a fuzzy neural network," Fuzzy Sets and Systems, vol. 73, no. 3, pp. 291-312, 1995.

[3] C. H. Lee and C. C. Teng, "Identification and control of dynamic systems using recurrent fuzzy neural networks," IEEE Transactions on Fuzzy Systems, vol. 8, no. 4, pp. 349-366, 2000.

[4] C. H. Lee and Y. C. Lin, "An adaptive type-2 fuzzy neural controller for nonlinear uncertain systems," Control and Intelligent Systems, vol. 33, no. 1, pp. 13-25, 2005.

[5] T. C. Lee, C. H. Wang, and H. L. Liu, “Observer-based adaptive fuzzy control for SISO nonlinear systems," Fuzzy Sets and Systems, vol. 148, no. 3, pp. 355-376, 2004.

[6] H. Li and S. Tong, "A hybrid adaptive fuzzy control for a class of nonlinear MIMO systems," IEEE Transactions on Fuzzy Systems, vol. 11, no. 1, pp. 24-34, 2003.

[7] Q. Liang and J. Mendel, "Interval type-2 fuzzy logic systems: theory and design," IEEE Transactions on Fuzzy Systems, vol. 8, no. 5, pp. 535-550, 2000.

[8] C. T. Lin and C. S. G. Lee, Neural Fuzzy Systems, Prentice Hall, Englewood Cliff, NJ, USA, 1996.

[9] F. J. Lin, R. J. Wai, and C. M. Hong, "Hybrid supervisory control using recurrent fuzzy neural network for tracking periodic inputs," IEEE Transactions on Neural Networks, vol. 12, no. 1, pp. 68-90, 2001.

[10] J. M. Mendel, Uncertain Rule-Based Fuzzy Logic Systems: Introduction and New Directions, Prentice-Hall, Englewood Cliff, NJ, USA, 2001.

[11] S. Tong and H. Li, "Fuzzy adaptive sliding-mode control for MIMO nonlinear systems," IEEE Transactions on Fuzzy Systems, vol. 11, no. 3, pp. 354-360, 2003.

[12] C. M. Kuan and K. Hornik, "Convergence of learning algorithms with constant learning rates," IEEE Transactions on Neural Networks, vol. 2, no. 5, pp. 484-489, 1991.

[13] R. H. Nielsen, "Theory of the backup-propagation neural network," in Proceedings of the International Joint Conference on Neural Networks (IJCNN' '89), vol. 1, pp. 593-605, 1989.

[14] C. H. Lee, "Stabilization of nonlinear nonminimum phase systems: adaptive parallel approach using recurrent fuzzy neural network," IEEE Transactions on Systems, Man, and Cybernetics, Part B: Cybernetics, vol. 34, no. 2, pp. 1075-1088, 2004.

[15] C. H. Wang, C. S. Cheng, and T. T. Lee, "Dynamical optimal training for interval type-2 fuzzy neural network (T2FNN)," IEEE Transactions on Systems, Man, and Cybernetics, Part B: Cybernetics, vol. 34, no. 3, pp. 1462-1477, 2004.

[16] X. H. Yu, G. A. Chen, and S. X. Cheng, "Dynamic learning rate optimization of the backpropagation algorithm," IEEE Transactions on Neural Networks, vol. 6, no. 3, pp. 669-677, 1995.

[17] L. A. Zadeh, "The concept of a linguistic variable and its application to approximate reasoning," vol. 8, pp. 199-249, 1975.

[18] J. M. Mendel, "Computing derivatives in interval type-2 fuzzy logic systems," IEEE Transactions on Fuzzy Systems, vol. 12, no. 1, pp. 84-98, 2004.

[19] J. M. Mendel, "Advances in type-2 fuzzy sets and systems," Information Sciences, vol. 177, no. 1, pp. 84-110, 2007.

[20] J. M. Mendel and R. I. B. John, “Type-2 fuzzy sets made simple," IEEE Transactions on Fuzzy Systems, vol. 10, no. 2, pp. 117-127, 2002.

[21] O. Castillo and P. Melin, Type-2 Fuzzy Logic: Theory and Applications, Springer, Berlin, Germany, 2008.

[22] O. Castillo and P. Melin, "A new approach for plant monitoring using Type-2 fuzzy logic and fractal theory," International Journal of General Systems, vol. 33, no. 2-3, pp. 305-319, 2004.

[23] O. Castillo, G. Huesca, and F. Valdez, "Evolutionary computing for optimizing type-2 fuzzy systems in intelligent control of non-linear dynamic plants," in Proceedings of the North American Fuzzy Information Processing Society (NAFIPS '05), pp. 247-251, Ann Arbor, Mich, USA, June 2005. 
[24] A. Van Ooyen and B. Nienhuis, "Improving the convergence of the back-propagation algorithm," Neural Networks, vol. 5, no. 3, pp. 465-571, 1992.

[25] J. J. E. Slotine and W. Li, Applied Nonlinear Control, Prentice-Hall, Upper Saddle River, NJ, USA, 1991.

[26] G. Chen and X. Dong, "On feedback control of chaotic continuous-time systems," IEEE Transactions on Circuits and Systems. I. Fundamental Theory and Applications, vol. 40, no. 9, pp. 591-601, 1993.

[27] G. Chen and X. Dong, "Identification and control of chaotic systems: an artificial neural network approach," in Proceedings of the IEEE International Symposium on Circuits and Systems (ISCAS '95), vol. 2, pp. 1177-1182, Seattle, Wash, USA, May 1995. 


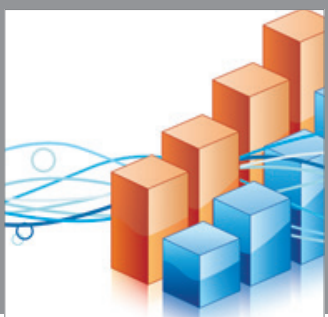

Advances in

Operations Research

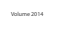

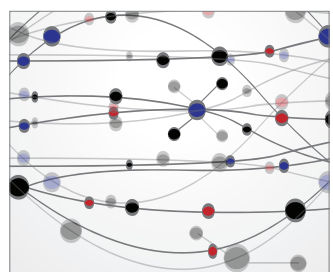

\section{The Scientific} World Journal
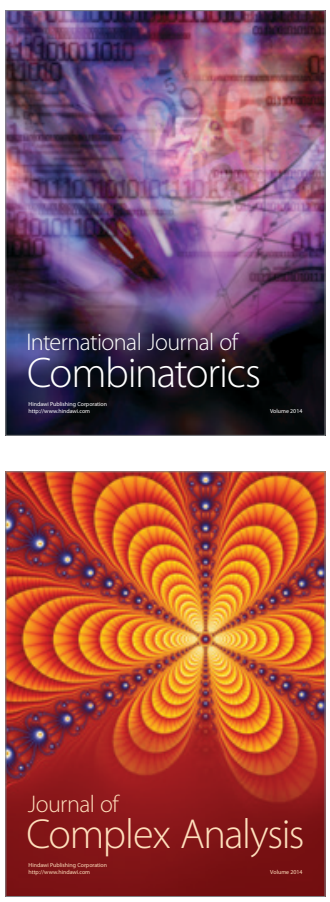

International Journal of

Mathematics and

Mathematical

Sciences
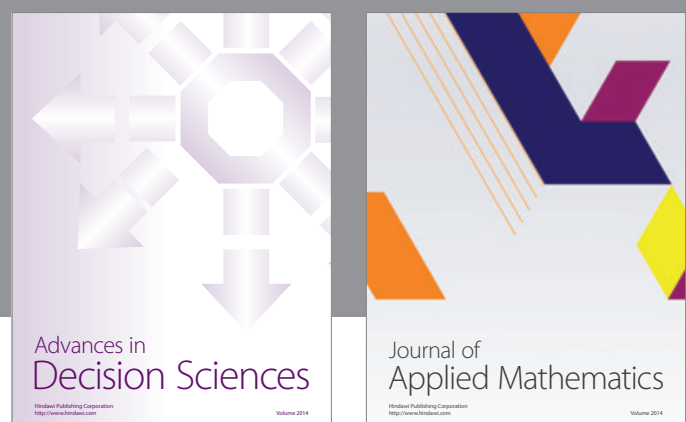

Journal of

Applied Mathematics
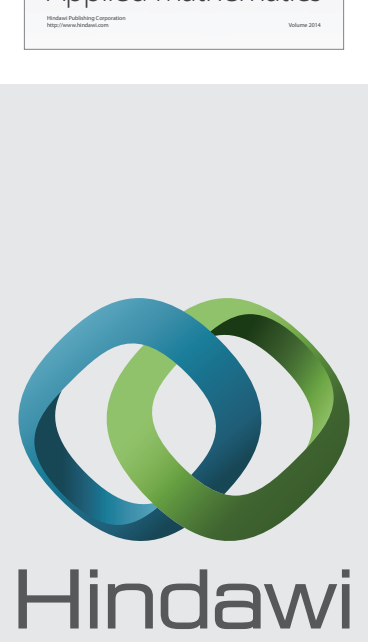

Submit your manuscripts at http://www.hindawi.com
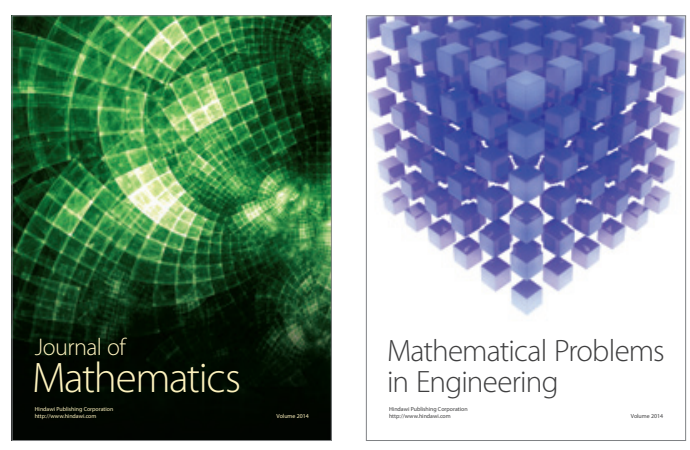

Mathematical Problems in Engineering
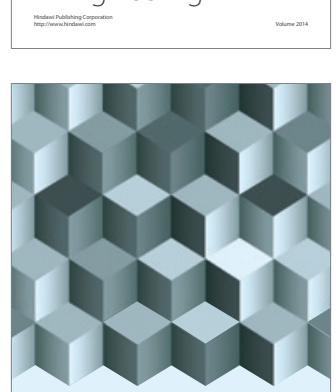

Journal of

Function Spaces
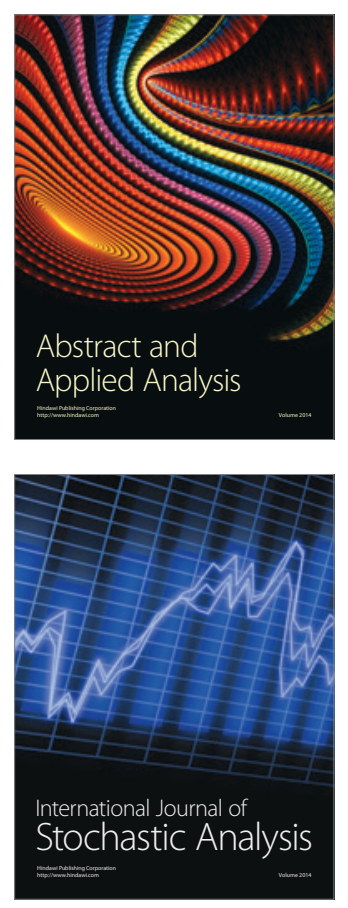

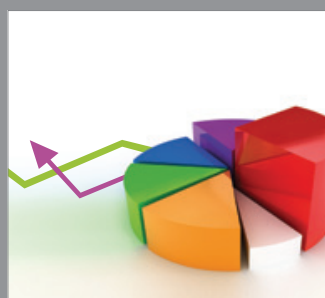

ournal of

Probability and Statistics

Promensencen
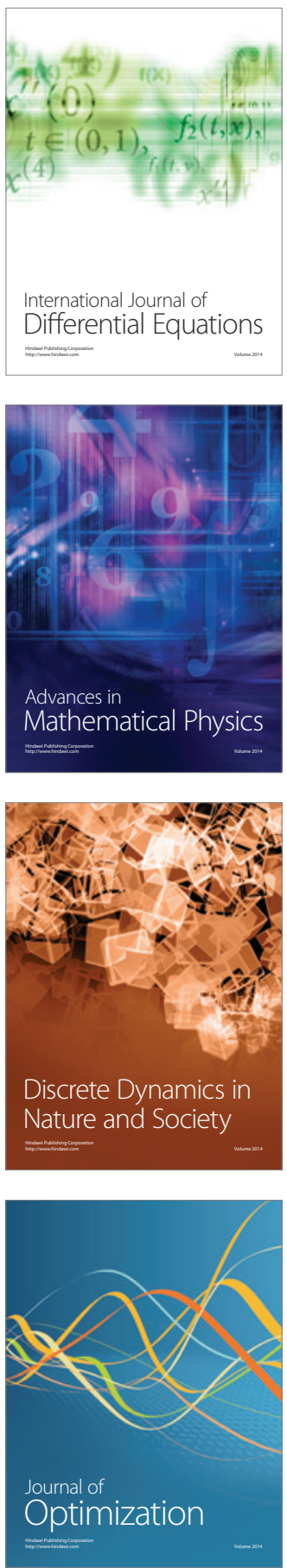